\title{
Intrinsic quantum Ising model on a triangular lattice magnet $\mathrm{TmMgGaO}_{4}$
}

\author{
Changle Liu, ${ }^{1,2,{ }^{*}}$ Chun-Jiong Huang $\odot, 1,3,4,5,{ }^{*}$ and Gang Chen $\oplus^{1,2,6,7, \dagger}$ \\ ${ }^{1}$ Department of Physics and HKU-UCAS Joint Institute for Theoretical and Computational Physics at Hong Kong, \\ The University of Hong Kong, Hong Kong, China \\ ${ }^{2}$ State Key Laboratory of Surface Physics and Department of Physics, Fudan University, Shanghai 200433, China \\ ${ }^{3}$ Shanghai Branch, National Laboratory for Physical Sciences at Microscale and Department of Modern Physics, University of Science and \\ Technology of China, Shanghai 201315, China \\ ${ }^{4}$ CAS Center for Excellence and Synergetic Innovation Center in Quantum Information and Quantum Physics, University of Science and \\ Technology of China, Hefei, Anhui 230026, China \\ ${ }^{5}$ CAS-Alibaba Quantum Computing Laboratory, Shanghai 201315, China \\ ${ }^{6}$ Collaborative Innovation Center of Advanced Microstructures, Nanjing University, Nanjing 210093, China \\ ${ }^{7}$ Institute of Nanoelectronics and Quantum Computing, Fudan University, Shanghai 200433, China
}

(Received 27 December 2019; revised 24 July 2020; accepted 15 September 2020; published 2 October 2020; corrected 11 December 2020)

\begin{abstract}
The rare-earth magnet $\mathrm{TmMgGaO}_{4}$ is proposed to be an intrinsic quantum Ising magnet described by the antiferromagnetic transverse field Ising model (TFIM) on a triangular lattice, where the relevant degrees of freedom are the nondegenerate dipole-multipole doublets of the $\mathrm{Tm}^{3+}$ ions and the transverse field has an intrinsic origin from the weak splitting of the doublet. We compare this special doublet of $\mathrm{Tm}^{3+}$ with the dipole-octupole Kramers doublet. We study the proposed effective model for the Tm-based triangular lattice and consider the effects of external magnetic fields and finite temperatures. From the orthogonal operator approach, we show that the TFIM with the three-sublattice intertwined ordered state agrees with the experiments and further clarify the discrepancy in the numbers of the magnetic sublattices and the measured magnon branches. We make specific predictions for the evolution of the magnetic properties with the external magnetic field. Furthermore, we demonstrate that an emergent U(1) symmetry emerges in thermal melting of the underlying orders and at the criticality, and summarize the previously known signatures related to the finite-temperature Berezinskii-Kosterlitz-Thouless physics. We discuss the broad relevance of intrinsic quantum Ising magnets to many other systems, especially Tm-based materials.
\end{abstract}

DOI: 10.1103/PhysRevResearch.2.043013

\section{INTRODUCTION}

Frustrated magnetism is an exciting field in modern condensed-matter physics and has been under active investigation for the past few decades. Generally speaking, frustration arises from competing interactions among local moments that cannot be satisfied simultaneously. The strong competition can give rise to exotic low-energy behaviors in frustrated magnets. This feature retains in the simplest classical antiferromagnetic Ising model, where for some particular frustrated lattices (triangular [1], Kagomé [2], pyrochlore $[3,4])$, there are macroscopic degenerate ground states associated with a finite zero-point entropy.

An interesting and important question to consider is the fate of classical macroscopic degeneracy in the presence of quantum fluctuations. Quantum fluctuations allow tunneling

\footnotetext{
*These two authors contributed equally to this paper.

†'gangchen@hku.hk

Published by the American Physical Society under the terms of the Creative Commons Attribution 4.0 International license. Further distribution of this work must maintain attribution to the author(s) and the published article's title, journal citation, and DOI.
}

within the macroscopic degenerate manifold, and therefore will lift the macroscopic degeneracy. Depending on lattice structures, the resulting quantum ground state can be either magnetically ordered or disordered [5-9], owing to the socalled order-by-disorder or disorder-by-disorder mechanism $[5,8,10-16]$. In practice, the simplest way to introduce quantum fluctuations is to add a transverse field to the Ising spins. The resulting model is the transverse field Ising model (TFIM), which has not only received considerable theoretical attention, but is also achievable in experiments. This model is sign-problem free in any lattice, therefore it can be efficiently dealt with by unbiased quantum Monte Carlo (QMC) simulations. These qualities render TFIM a good platform for collaborations among experimental, theoretical, and numerical communities.

In realistic materials, two distinct physical origins of the transverse field were proposed and have been summarized in Ref. [17]. These two distinct ones are referred to as extrinsic origin and intrinsic origin. For the extrinsic origin, the transverse spin components act as ordinary magnetic dipole moments, hence the transverse field is directly achievable with the physical magnetic field along the transverse directions. This mechanism applies to various $\mathrm{Co}$ based Ising magnets such as $\mathrm{CoNb}_{2} \mathrm{O}_{6}$ [18-20], $\mathrm{BaCo}_{2} \mathrm{~V}_{2} \mathrm{O}_{8}$ [21-23], and $\mathrm{SrCo}_{2} \mathrm{~V}_{2} \mathrm{O}_{8}$ [24,25]. For the intrinsic origin, the 
transverse field is generated internally and models the intrinsic crystal-field splitting between two relevant crystal-field levels that are responsible for the low-temperature magnetism. It was further proposed that rare-earth magnets with low crystal-field symmetries would automatically generate such an intrinsic transverse field for the local moments with even number of electrons. This is because the low crystal-field symmetries cannot provide enough symmetry operations that protect the degeneracy of the crystal-field levels. Nevertheless, the intrinsic transverse field could also emerge in the case with high crystal-field symmetries. This was emphasized for $\mathrm{TmMgGaO}_{4}$ in the introduction of Ref. [17] as an example of the intrinsic transverse field.

The TFIM with an intrinsic transverse field was first proposed for $\mathrm{TmMgGaO}_{4}$ in Ref. [26]. $\mathrm{TmMgGaO}_{4}$ [27-29] is a Mott insulator in which the $\mathrm{Tm}^{3+}$ ions form a perfect triangular lattice. Experimentally, thermodynamic [27-29] and detailed neutron-scattering [26] measurements have been performed, and the elementary spin-wave-like excitation spectrum with respect to the magnetically ordered ground state has been well recorded [26]. In this system, the two lowest crystal field levels of the $\mathrm{Tm}^{3+}$ ion that contribute to the local moment are the point-group-symmetry-demanded singlets. This intrinsic transverse field arises from the intrinsic splitting between the two singlets. The crystal field splitting is demanded by symmetry and appears at the atomic level, so it cannot be ignored compared to exchange interactions and must be considered in the first place. This is explained in detail in Secs. II and III. Moreover, in $\mathrm{TmMgGaO}_{4}$ the transverse and longitudinal spin components behave fundamentally different in nature; the system exhibits antiferromagnetic dipolar order coexisting with the preformed multipolar order due to the intrinsic transverse field [26]. The resulting state is an example of the intertwined multipolar order, originally proposed in the context of non-Kramers doublet systems in rare-earth magnets [30], and also applies for $\mathrm{TmMgGaO}_{4}$.

In this paper, we systematically explore our proposed TFIM for $\mathrm{TmMgGaO}_{4}$ and understand the physics of the Tmbased triangular lattice antiferromagnets from a combination of techniques and perspectives that involve the microscopics, the thermodynamic and neutron scattering experiments, the many-body modeling, the QMC simulation and meanfield analysis, and the connection between the theory and the measurements. Our effort in this paper requires a sophisticated blending and mutual feedback amongst the microscopic physics, the many-body physics, and the experimental understanding. Therefore, this paper does not have a single thread of logic flow in the organization of the sections. To guide the readers well, we outline the content of the remaining parts of the papers here. In Sec. II, we explain the nondegenerate nature of the two lowest crystal field levels of the $\mathrm{Tm}^{3+}$ ion and refer them as the nondegenerate dipole-multipole (DM) doublet. We further compare the Tm-based nondegenerate DM doublet with the well-known dipole-octupole (DO) doublet. In Sec. III, we explore the symmetry properties of the effective spin operators, write down the TFIM for $\mathrm{TmMgGaO}_{4}$, and review the experiments. In Sec. IV, we combine mean-field calculation, QMC simulation, and theoretical arguments to establish the finite temperature phase diagram of our proposed TFIM on the triangular lattice. We explore the thermal

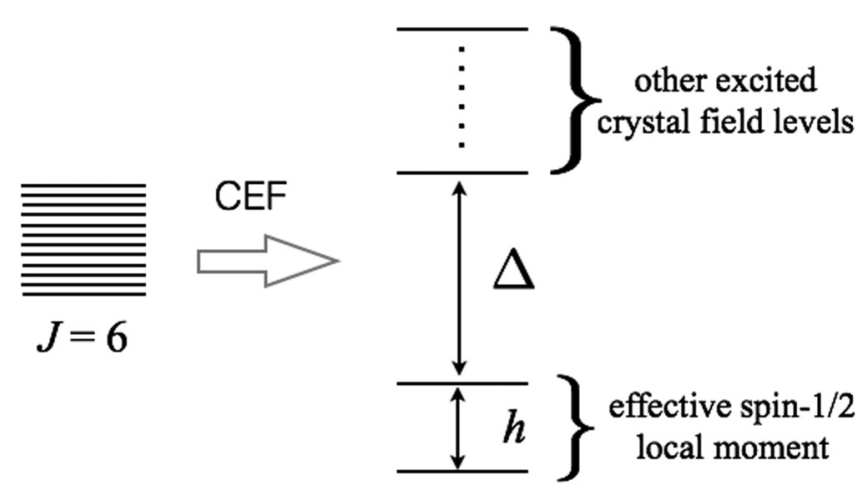

Local moment of the $\mathrm{Tm}^{3+}$ ion

FIG. 1. The splitting of the $J=6$ total moment of the $\mathrm{Tm}^{3+}$ ion in $\mathrm{TmMgGaO}_{4}$ under the $\mathrm{D}_{3 d}$ crystal electric field (CEF). The energy separation, $h$, between the ground-state singlet and the first excited singlet is much smaller than the energy gap, $\Delta$, to the other excited crystal-field levels, and the two lowest levels are responsible to the low-temperature magnetic properties.

Berezinskii-Kosterlitz-Thouless (BKT) phase and transitions, as well as the emergent continuous U(1) symmetry near the transitions. In Sec. V, we apply the orthogonal operator approach to explain the selective measurements. From this understanding, we were able to establish the connection between the theoretical results and the experiments. We establish the magnetic excitations in different phases and point out the qualitative differences between them. We clarify the the discrepancy between the magnetic sublattices and the branches of the measured magnon excitations in the ordered side. In Sec. VI, we explore the effect of the external magnetic fields in various physical quantities. We show the nonmonotonic behaviors of the magnetic Bragg peak in magnetic fields, the evolution of the magnetic excitation with the fields, and the thermodynamic behaviors. In Sec. VII, we summarize our understanding about $\mathrm{TmMgGaO}_{4}$ and point out the relevance of the intrinsic TFIM for other Tm-based magnets. In the Appendix, we provide the results from the linear spin-wave theory where the full structures of the magnetic excitations are available. These features are compared with the results from the selective measurements.

\section{NONDEGENERATE DIPOLE-MULTIPOLE DOUBLET OF $\mathrm{Tm}^{3+}$ ion}

For completeness, we explain the microscopics of the $\mathrm{Tm}^{3+}$ [26] in $\mathrm{TmMgGaO}_{4}$. The $\mathrm{Tm}^{3+}$ ion has a total orbital angular moment $L=5$ and total spin moment $S=1$, and the spin-orbit coupling gives a total moment $J=6[26,28]$. The 13-fold degeneracy of the $J$ moment is split by the crystal field. The ground state and the first excited state of $\mathrm{Tm}^{3+}$ are both singlets (see Fig. 1) and are well separated from other excited levels. These two states together are dubbed DM doublet and are given as a linear superposition of $\left|J^{z}=3 n\right\rangle$ with

$$
\begin{gathered}
\left|\Psi_{g}\right\rangle=c_{6}[|6\rangle+|-6\rangle]+c_{3}[|3\rangle-|-3\rangle]+c_{0}|0\rangle, \\
\left|\Psi_{e}\right\rangle=c_{6}^{\prime}[|6\rangle-|-6\rangle]+c_{3}^{\prime}[|3\rangle+|-3\rangle],
\end{gathered}
$$


(a)

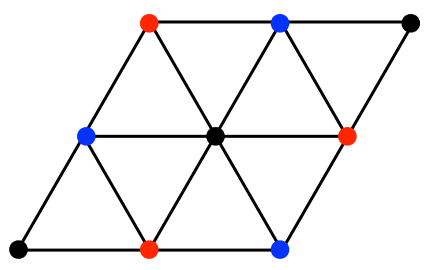

(b)

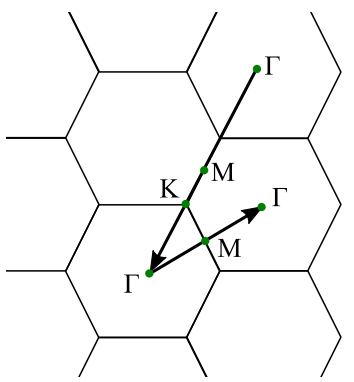

FIG. 2. (a) Definition of the triangular lattice. The three sublattices are marked in blue, red, and black, respectively. (b) The Brillouin zone of the triangular lattice.

where $|3 n\rangle$ (with $n \in \mathbb{Z}$ ) refers to the quantum number of $J^{z} .\left|\Psi_{g}\right\rangle\left(\left|\Psi_{e}\right\rangle\right)$ refers to the ground state (the first excited crystal-field level), and the two singlets carry $A_{1 g}$ and $A_{2 g}$ representation of the $D_{3 d}$ point group, respectively. Here $c_{6}, c_{3}, c_{0}, c_{6}^{\prime}, c_{3}^{\prime}$ are real numbers with $\left|c_{6}\right| \approx\left|c_{6}^{\prime}\right| \gg c_{3}, c_{3}^{\prime}, c_{0}$. Their nature of the one-dimensional (1D) irreducible representation can be simply seen by applying the threefold rotation operation:

$$
e^{-i \frac{2 \pi}{3} J^{z}}\left|J^{z}=3 n\right\rangle=\left|J^{z}=3 n\right\rangle .
$$

Other integer spin numbers do not have this property, and they often give rise to two-dimensional (2D) representations of the $\mathrm{D}_{3 d}$ point group. Due to the integer spin nature for $\mathrm{Tm}^{3+}$, there is no Kramers-theorem-demanded degeneracy.

Note that both $\left|\Psi_{g}\right\rangle$ and $\left|\Psi_{e}\right\rangle$ are nonmagnetic, and thus thinking locally about the single-ion physics would not lead to any magnetism. The magnetism should come from the exchange interaction between the local moments. The intrinsic competition between the single-ion physics and the exchange is captured and modeled as an intrinsic TFIM [26] and will be explained in Sec. III.

As the $\mathrm{Tm}^{3+} \mathrm{DM}$ doublet here was sometimes referred to as a non-Kramers doublet, we clarify their difference. The usual non-Kramers doublet that occurs in, for example, the $\mathrm{Pr}^{3+}$ ion $[31,32]$ of $\operatorname{Pr}_{2} \mathrm{Zr}_{2} \mathrm{O}_{7}$ and $\mathrm{Pr}_{2} \mathrm{Ir}_{2} \mathrm{O}_{7}$ or other rare-earth triangular lattice magnets [30], is composed of two degenerate crystal-field states, and their degeneracy is not protected by time reversal but protected by the point-group symmetry. These states comprise the 2D irreducible representation. In comparison, the $\mathrm{Tm}^{3+}$ doublet are two singlets with two independent 1D irreducible representations.

It is instructive to compare the DM doublet of $\mathrm{Tm}^{3+}$ with the DO doublet that also arises from the 1D irreducible representations of the $\mathrm{D}_{3 d}$ point group [30,33-36] (see Table I). For the DO doublet, the wave function of each state in the doublet is a linear superposition of $\left|J^{z}=3 n / 2\right\rangle$ where $n$ is an odd integer. The DO doublet was found to be applicable to $\mathrm{Nd}^{3+}$ in Nd-based pyrochlores [37-44], $\mathrm{Sm}^{3+}$ in $\mathrm{Sm}_{2} \mathrm{Ti}_{2} \mathrm{O}_{7}[45,46]$, $\mathrm{Ce}^{3+}$ in $\mathrm{Ce}_{2} \mathrm{Sn}_{2} \mathrm{O}_{7}$ [34,47,48], and $\mathrm{Ce}_{2} \mathrm{Zr}_{2} \mathrm{O}_{7}$ [49-51] and $\mathrm{Er}^{3+}$ in the spinels $[52,53]$. DO doublets can broadly exist in magnets with a $\mathrm{D}_{3 d}$ point-group symmetry, other lattices such as honeycomb magnets could support the DO doublet [54]. As a parallel thought, the $\mathrm{Tm}^{3+} \mathrm{DM}$ doublet could broadly exist in many other structures (see Sec. VII).

TABLE I. The comparison between the nondegenerate dipolemultipole (DM) doublet of $\mathrm{Tm}^{3+}$ and the dipole-octupole (DO) doublet for Kramers ions.

\begin{tabular}{lcc}
\hline \hline Properties & Nondegenerate DM doublet & DO doublet \\
\hline original moment & integer & half-odd integer \\
time reversal & $S^{z} \rightarrow-S^{z}$ & $S^{z} \rightarrow-S^{z}$ \\
time reversal & $S^{x, y} \rightarrow S^{x, y}$ & $S^{x, y} \rightarrow-S^{x, y}$ \\
degeneracy & two separate singlets & 2-fold degenerate \\
threefold rotation & eigenvalue +1 & eigenvalue -1 \\
\hline \hline
\end{tabular}

\section{EFFECTIVE MODEL OF TmMgGaO $\mathrm{T}_{4}$}

Like any two-level systems, the $\mathrm{Tm}^{3+} \mathrm{DM}$ doublet can be captured by effective spin- $1 / 2$ operators that operate on the doublet. We define the effective spin-1/2 operator $S_{i}$ on each Tm site as

$$
\begin{aligned}
S_{i}^{x} & =\frac{i}{2}\left(\left|\Psi_{i, e}\right\rangle\left\langle\Psi_{i, g}|-| \Psi_{i, g}\right\rangle\left\langle\Psi_{i, e}\right|\right), \\
S_{i}^{y} & =\frac{1}{2}\left(\left|\Psi_{i, g}\right\rangle\left\langle\Psi_{i, g}|-| \Psi_{i, e}\right\rangle\left\langle\Psi_{i, e}\right|\right), \\
S_{i}^{z} & =\frac{1}{2}\left(\left|\Psi_{i, g}\right\rangle\left\langle\Psi_{i, e}|+| \Psi_{i, e}\right\rangle\left\langle\Psi_{i, g}\right|\right) .
\end{aligned}
$$

One sees from the definition that $\left|\Psi_{i, g / e}\right\rangle$ are eigenstates of $S^{y}$, while the $S^{x}$ and $S^{z}$ introduce hybridization between $\left|\Psi_{i, g / e}\right\rangle$. The point-group symmetry demanded a splitting between $\left|\Psi_{i, g}\right\rangle$ and $\left|\Psi_{i, e}\right\rangle$ is modeled as an intrinsic transverse field on $S^{y}$, i.e., $-h \sum_{i} S_{i}^{y}$, where $h$ is the splitting. Moreover, the $x$ and $y$ in $S^{x}$ and $S^{y}$ are defined in the internal Hilbert space of the DM doublet, $\left|\Psi_{i, g}\right\rangle$ and $\left|\Psi_{i, e}\right\rangle$, and have no connection to the real space. Nevertheless, we often refer to these two components as in-plane components for convenience. $S^{z}$ has its physical meaning both for the real space and for the internal Hilbert space. Under the time reversal $(\mathcal{T})$, the effective spin transforms as

$$
\mathcal{T}: \quad S_{i}^{x} \rightarrow+S_{i}^{x}, S_{i}^{y} \rightarrow S_{i}^{y}, S_{i}^{z} \rightarrow-S_{i}^{z} .
$$

$S^{x}$ and $S^{y}$ are even under $\mathcal{T}$ and transform as even-order multipoles under the crystal symmetries. From $\left|\Psi_{g}\right\rangle$ and $\left|\Psi_{e}\right\rangle$, it is clear that $S^{x}$ and $S^{y}$ mostly connect $\left|J^{z}=6\right\rangle$ and $\left|J^{z}=-6\right\rangle$ and mostly involve the 12th-order multipole moments. $S^{z}$ is odd under $\mathcal{T}$ and transforms as a dipole moment. The low-temperature magnetization is provided by $\left\langle\sum_{i} S_{i}^{z}\right\rangle$. While the dipole moment, $S^{z}$, is probed by neutron scattering, the multipole moments are hidden or invisible in conventional probes and are often referred to as hidden orders or hidden components in the literature.

From the saturated moment values in the magnetic field, one infers that the Tm local moment is almost an Ising spin. This is also understood from the wave functions of $\left|\Psi_{g}\right\rangle$ and $\left|\Psi_{e}\right\rangle$ where $\left|J^{z}= \pm 6\right\rangle$ are dominant. The exchange between the Tm local moments would primarily be an Ising interaction. The exchange between the transverse components are strongly suppressed as $S^{x}$ and $S^{y}$ are high order multipoles and they 
are even higher than the quadrupoles. The resulting effective Hamiltonian for the interacting Tm local moment is the TFIM

$$
H=\sum_{\langle i j\rangle} J_{z z} S_{i}^{z} S_{j}^{z}-\sum_{i}\left(h S_{i}^{y}+B S_{i}^{z}\right)
$$

where $B \equiv \mu_{B} g_{\|} B^{z}$ represents the external magnetic field along the $z$ direction and spins locate on a triangular lattice, as shown in Fig. 2. In Ref. [26], we actually included a tiny second-neighbor Ising interaction $J_{2}$ to improve the fitting to the experiments. As the interaction energy scale between the Tm local moment is already quite small, the tiny $J_{2}$ does not change the qualitative physics in this paper. Thus, we rely on the above minimal model to capture the essential physics about $\mathrm{TmMgGaO}_{4}$ and other intrinsic quantum Ising magnets. Nevertheless, if one is more interested in the quantitative aspects, other nonessential and nonuniversal ingredients should be included into our Hamiltonian. These would involve the long-range dipole-dipole $\left(S^{z}-S^{z}\right)$ interaction and the Van Vleck process through the excited crystal-field states.

The $\mathrm{Tm}^{3+}$ magnetic moment is much larger than the one for the $\mathrm{Yb}^{3+}$ ion in $\mathrm{YbMgGaO}_{4}$. Although the firstneighbor dipole-dipole interaction may be incorporated with the superexchange interaction and modelled as a total $J_{z z}$ interaction, we here estimate the further neighbor dipole-dipole interaction and find that the second-neighbor dipole-dipole interaction is $0.48 \mathrm{~K}$, the third neighbor is $0.31 \mathrm{~K}$, the fourth neighbor is $0.134 \mathrm{~K}$, the fifth neighbor is $0.092 \mathrm{~K}$, and the sixth neighbor is $0.053 \mathrm{~K}$. The Curie-Weiss temperature is $(-19 \mathrm{~K})$ [26] and was obtained from fitting the lowtemperature magnetic susceptibility without subtracting the Van Vleck contribution. If one simply attributes all the further neighbor interactions beyond the first neighbor to the dipoledipole interactions, one readily finds that the first-neighbor interaction $J_{z z}$ is $\sim 11.5 \mathrm{~K}$ and should dominate over further neighbor interactions. Thus, it is legitimate for us to keep only the first neighbor or first few neighbors in TFIM. Moreover, the energy gap from the doublet to the lowest crystal-field excited level is smaller than the one in $\mathrm{YbMgGaO}_{4}$ [55]. Thus the virtual Van Vleck process could further bring extra ingredients into the quantitative modeling.

Here we briefly review the previous experimental efforts. To the best of our knowledge, the single crystal sample of $\mathrm{TmMgGaO}_{4}$ and its basic structure and thermodynamic properties were reported in Ref. [27]. Even though the measurements were performed above $1.8 \mathrm{~K}$, the magnetization results already show the strong Ising-like features. More lowtemperature thermodynamic measurements were obtained in Ref. [28], and the results were interpreted from classical Ising moments with competing Ising interactions. The lowtemperature magnetic state was suggested to be a stripe order with an alternating Ising spin arrangement on two magnetic sublattices, and the transition to the stripe order was suggested to occur at $\sim 0.27 \mathrm{~K}$. This spin state has an ordering wave vector at $M$ point in the Brillouin zone. The detailed elastic and inelastic neutron scattering measurements were performed in Ref. [26] together with the low-temperature thermodynamic measurements. The appearance of the magnetic Bragg peak at $K$ point coincides with the peak at $\sim 1 \mathrm{~K}$ in the specific heat data. The ordering wave vector $K$ indicates a three-

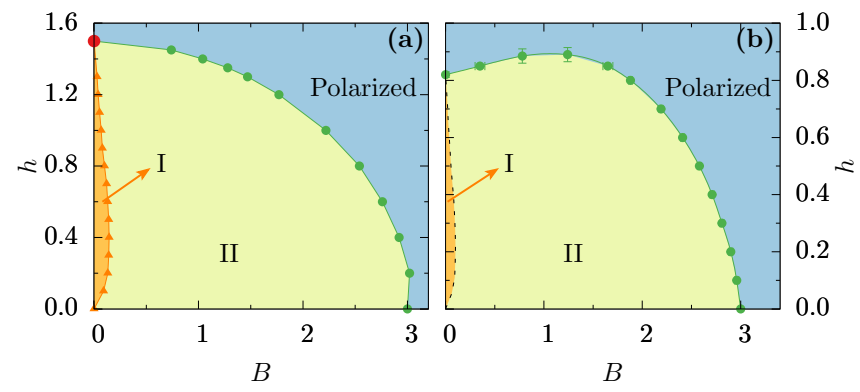

FIG. 3. Phase diagram of the model in Eq. (8). Here we set the energy unit $J_{z z}=1$. Two three-sublattice ordered phases, I (orange) and II (green), and a polarized phase are found in the phase diagram. The red dot represents quantum phase transition with $(2+1) \mathrm{d} X Y$ universality class. The left (a) is the mean-field result and the right (b) is the Monte Carlo result that is calculated at inverse temperature $\beta=8 L$ with system sizes $N=L \times L(L=6,12,24)$. The phase boundary from phase I to II is difficult to capture in the current algorithm and are schematic here.

sublattice magnetic order, which differs from the proposal of stripe order in Ref. [28]. Moreover, the data-rich inelastic neutron-scattering measurements show a coherent spin-wavelike excitation with a well-defined dispersion.

To reconcile these experiments and the DM nature of the $\mathrm{Tm}^{3+}$ local moments, one concludes with an intertwined dipolar and multipolar orders for the ground state of $\mathrm{TmMgGaO}_{4}$ [26],

$$
\begin{gathered}
\left\langle S^{z}\right\rangle \neq 0 \quad \text { with a three-sublattice structure } \\
\left\langle S^{x}\right\rangle \neq 0 \quad \text { and } / \text { or } \quad\left\langle S^{y}\right\rangle \neq 0 .
\end{gathered}
$$

From the microscopics and our modeling, it is obvious to see that the invisible component, $S^{y}$, is nonzero as it is polarized by the intrinsic transverse field. In next few sections, we will examine whether our modeling can provide useful understanding of the physical properties and insights for future experiments on $\mathrm{TmMgGaO}_{4}$ and/or other Tm-based triangular lattice magnets.

\section{PHASE DIAGRAM}

\section{A. Mean-field analysis}

The TFIM on the triangular lattice has been well studied in the absence of the external magnetic field [6,56], while the situation with the longitudinal field has not been investigated yet. To gain some physical insight into the ground-state phase diagram, we first tackle with the Weiss mean-field approximation by decoupling interactions between different spins as

$$
S_{i}^{z} S_{j}^{z} \rightarrow\left\langle S_{i}^{z}\right\rangle S_{j}^{z}+S_{i}^{z}\left\langle S_{j}^{z}\right\rangle-\left\langle S_{i}^{z}\right\rangle\left\langle S_{j}^{z}\right\rangle .
$$

Here the mean-field order parameter $\left\langle S_{i}^{z}\right\rangle$ needs to be solved self-consistently. The mean-field phase diagram is depicted in Fig. 3(a).

In the Ising limit (without the transverse and longitudinal fields), the system lies at a classically critical state that hosts a macroscopic ground-state degeneracy: Any spin configuration with 2-up-1-down or 1-up-2-down has the minimal energy. With introducing the transverse field $h$, quantum fluctuations allow quantum tunneling within the massively degenerate 
manifold. This quantum tunneling lifts the macroscopic degeneracies and eventually stabilizes a three-sublattice longrange ordered phase (dubbed the three-sublattice I state) as the ground state owing to the quantum order-by-disorder mechanism. Since the three-sublattice ordering is entirely contributed by the quantum fluctuations, it is relatively weak and is controlled by quantum fluctuation $h$ in a nonmonotonic fashion: With $h$ being too small, the quantum order-bydisorder effect is weak, while for a very large $h$ the polarization effect becomes more important, suppresses the threesublattice ordering, and drives the system into the "quantum disordered" state where the spins are fully polarized along the transverse direction. Although the above results are obtained mean-field level, they are consistent with those obtained via quantum dimer model mapping where quantum fluctuations are taken into account in a perturbative manner $[5,6]$.

As the external longitudinal field $B$ is applied at the Ising limit, the system immediately becomes unstable against the magnetic ordering due to the criticality at this point. The resulting state is another three-sublattice ordered state called 1/3-plateau state with a 2-up-1-down structure on each triangular plaquette. Unlike the pure quantum origin in the I phase, the three-sublattice ordering of the plateau state arises at the classical level and are more stable. The plateau state remains as the ground state upon increasing the magnetic field until the system becomes fully polarized at $B_{c}=3 J_{z z}$ through a first-order transition. When the quantum fluctuation $h$ is switched on, the three-sublattice plateau state becomes the quasiplateau phase (dubbed three-sublattice II state) because the total magnetization is no longer a good quantum number. Moreover, as the three-sublattice I phase is generated by the quantum fluctuations and is fully gapped, it is stable against the weak perturbations. But since that the ordering is rather weak, a small external field $B$ could drive the system to the quasiplateau state across a phase transition. The transition from the I to II state is of the second order, while the transition from II to the fully polarized state is of the first order, consistent with what happens at $h=0$ limit. The two phase boundaries terminate at the classical critical point $h=0$, and at the quantum critical point $h_{c}^{\mathrm{MF}}=1.5 J_{z z}$, both located along the $B=0$ axis. These are depicted in Fig. 3(a) and obtained from the mean-field analysis.

\section{B. Path-integral quantum Monte Carlo method}

To examine our mean-field results, we perform the QMC simulations. We choose the the path integral with the $\left\{S_{i}^{z}\right\}$ basis. The partition function of the original model is mapped onto a world-line representation:

$$
\begin{aligned}
\mathcal{Z} & =\operatorname{Tr}\left[e^{-\beta \mathcal{H}}\right]=\sum_{\left\{\alpha_{0}\right\}}\left\langle\alpha_{0}\left|e^{-\beta \mathcal{H}}\right| \alpha_{0}\right\rangle \\
& =\lim _{\substack{d \tau=\frac{\beta}{3} \\
n \rightarrow \infty}} \sum_{\substack{\{\alpha\} \\
\alpha_{n}=\alpha_{0}}}\left\langle\alpha_{n}\left|e^{-\mathcal{H} d \tau}\right| \alpha_{n-1}\right\rangle \cdots\left\langle\alpha_{1}\left|e^{-\mathcal{H} d \tau}\right| \alpha_{0}\right\rangle \\
& =\sum_{\{\alpha\}} \sum_{k=0}^{\infty} \int_{0}^{\beta} \cdots \int_{\tau_{2 k-1}}^{\beta} \prod_{i=1}^{2 k} d \tau_{i} h^{2 k} e^{-\int_{0}^{\beta} U(\tau) d \tau},
\end{aligned}
$$
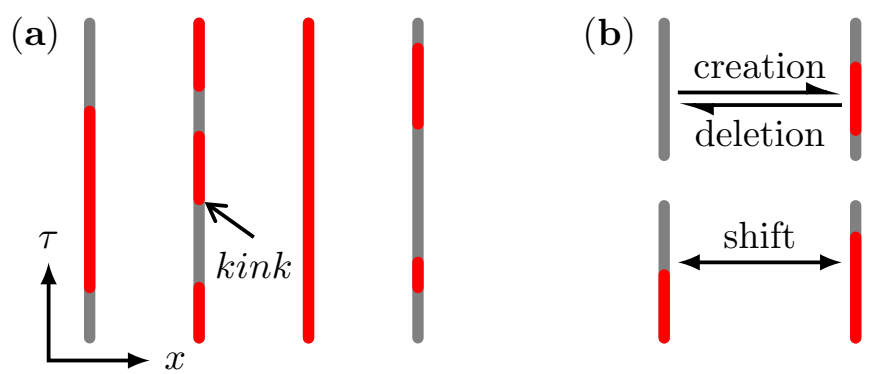

FIG. 4. The world-line configuration under imaginary time evolution and update schemes. (a) is a world-line configuration of four spins in a chain. Different colors correspond to different spin $S^{z}$ states. Along the imaginary time, every spin world line may be divided into several flats by cause of $S_{i}^{y}$. (b) the diagrammatic sketch of the update schemes.

where

$$
U(\tau)=\left\langle\alpha(\tau)\left|\left(\sum_{\langle i j\rangle} J_{z z} S_{i}^{z} S_{j}^{z}-\sum_{i} B S_{i}^{z}\right)\right| \alpha(\tau)\right\rangle .
$$

In Fig. 4(a), we depict a representative world-line configuration that contributes to the partition function. The transverse field term of $\sum_{i} h S_{i}^{y}$ causes the spin $S^{z}$ to flip, and we refer to such a flipping event as a kink. The temporal periodic boundary condition $|\alpha(0)\rangle=|\alpha(\beta)\rangle$ of the path integral demands the number of the kinks $N_{k}$ to be even with $N_{k}=2 k(k \in \mathbb{Z})$ in Eq. (12). Due to the presence of the longitudinal field $B$, the cluster update fails, and instead, we design a metropolis algorithm that contains two update schemes, creation/deletion flat and shift kink, as shown in Fig. 4. The calculations of acceptance rates of update schemes are quite standard through the detailed balance equation and we will not show them explicitly here. The thermal annealing procedure is employed to deal with the freezing issue of the Monte Carlo simulation.

In the QMC simulations, we take the system sizes $N=L \times L(L=6,12,24)$ with periodic boundary conditions. The ground-state phase diagram is calculated at inverse temperature $\beta=8 L$ and the result is shown in Fig. 3(b) through the finite-size scaling. The QMC phase diagram agrees with the mean-field one at the qualitative level. The locations of the phase boundaries differ quantitatively. The critical field $h_{\mathrm{cC}}^{\mathrm{MC}} \approx 0.82 J_{z z}[56,57]$ is almost half of the meanfield result $h_{c}^{\mathrm{MF}}=1.5 J_{z z}$ with zero external field $B=0$. This is as expected, as the mean-field approximation underestimates the quantum fluctuations, especially for the phase boundaries. Nevertheless, the mean-field theory provides the essential physical understanding and insights for the magnetic properties of the system.

\section{Finite temperature regimes and BKT transitions}

In this subsection, we extend the analysis from the zero temperature or the near-ground-state low temperatures to finite temperatures and study the finite temperature properties and the phase transitions out of the ordered one. To reveal the finite-temperature transitions, it is necessary to perform the field theoretical analysis near the transition and then supplement with the QMC calculations. The three-sublattice order 

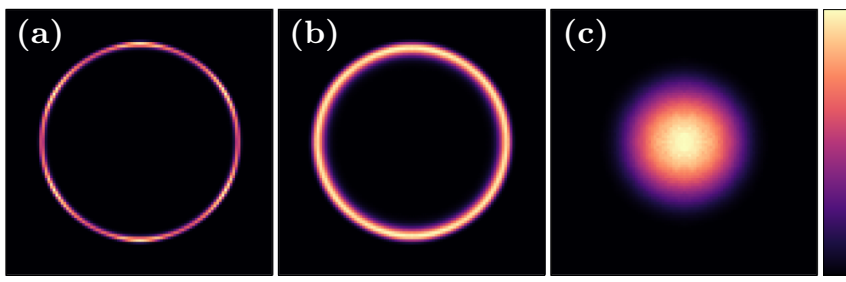

FIG. 5. Histograms of the order parameter $\psi$ in different temperature regimes obtained from QMC simulations. (a) Threesublattice long-range ordered state at low temperature $T=0.025$. (b) Quasi-long-range ordered BKT phase at intermediate temperature $T=0.075$. (c) Disordered state at high temperature $T=0.225$. In the QMC simulations, we set the model parameter $J_{z z}=1, h=0.4$, $B=0$, and the system size $L=12$.

parameter is characterized by the Fourier transformed $S^{z}$ dipolar component at the $K$ point. This can be captured by the following complex field:

$$
\psi=\frac{1}{\sqrt{3}}\left(m_{1}+m_{2} e^{i 2 \pi / 3}+m_{3} e^{-i 2 \pi / 3}\right),
$$

where $m_{i}(i=1,2,3)$ are the dipolar magnetizations of the three sublattices at the neighboring sites and we have set the lattice constant to unity. We can see that $\psi$ characterizes the three-sublattice ordering, as $\psi=0$ occurs only when $m_{1}=m_{2}=m_{3}$, where the three-sublattice order vanishes. The transformation of the field variable $\psi$ under the lattice translation $T_{\hat{x}}$ and the time reversal $\mathcal{T}$ operation take the following form:

$$
\begin{gathered}
T_{\hat{x}}: \psi \rightarrow \psi e^{i 2 \pi / 3}, \\
\mathcal{T}: \psi \rightarrow-\psi .
\end{gathered}
$$

For the three-sublattice I state, the spin alignments at the three sublattices are different from one another, therefore the ground state is sixfold degenerate. With zero external magnetic field, the $\psi$ corresponding to the ground states are located at a circle in the complex plane with $\operatorname{Arg} \psi=(2 n+1) \pi / 6(n=0,1, \ldots, 5)$ that are protected by the translation and time-reversal symmetry [see Fig. 5(a)]. This clock anisotropy is robust against the short-range interactions such as weak transverse exchange and nextnearest-neighbor Ising interactions that are present in the materials, therefore our analysis remains valid against these perturbations. In the vicinity of the melting of the magnetic order, the coarse-grained Landau-Ginzburg-Wilson free energy dictates the $\mathbb{Z}_{6}$ clock anisotropy and takes the following form [58]:

$$
\begin{aligned}
H_{\mathrm{LGW}}= & -K|\nabla \psi|^{2}+r \psi^{*} \psi+u_{4}\left(\psi^{*} \psi\right)^{2}+u_{6}\left(\psi^{*} \psi\right)^{3} \\
& +v_{6}\left(\psi^{6}+\psi^{* 6}\right)
\end{aligned}
$$

with $\psi=|\psi| e^{i \theta}$, where $\theta$ corresponds to the phase of the field $\psi$. The $\mathbb{Z}_{6}$ clock anisotropy term $v_{6}$ has a significant implication on the nature of thermal and quantum phase transitions. First, let us examine the thermal melting of the three-sublattice states. Since the clock anisotropy term is brought about by the quantum fluctuations from the transverse field and is expected to be small, the phase fluctuations of the order parameter $\psi$ is soft and therefore becomes important for the thermal melting at the first stage. By integrating out the amplitude fluctuations, we obtain the 2D XY model with a $\mathbb{Z}_{6}$ clock anisotropy. This theory exhibits an approximate self-duality $[59,60]$, where the dual theory is described in terms of vortices of $\theta$ that act as the disorder parameter of the original theory. It was previously understood that certain self-dual quantum critical points can put constraints on the physical observables such as a nondivergent Grüneisen ratio [61]. The current transition is an approximate self-duality and is driven by temperature. Whether an analogous property can occur here will be explored in future work.

The thermal melting of the three-sublattice order takes a two-step manner $[5,56,62,63]$ and is also clearly identified in the order parameter histogram as is shown in Fig. 5. At the low-temperature phase $T<T_{c 1}$ that is proximate to the ground state, the $\mathbb{Z}_{6}$ clock term is relevant such that the phase of $\phi$ is pinned to six equivalent angles, and we have the three-sublattice long-range ordered state. This can be seen in the angular histogram plot of the order parameter $\psi$ in Fig. 5(a) where the sixfold variation is shown. The dual phase at $T>T_{c 2}$ is the high-temperature disordered phase where the vortices proliferate. The higher temperature transition at $T_{c 2}$ belongs to the BKT universality class, while the lower temperature transition at $T_{c 1}$ is dual to the high temperature one and hence is called the inverse BKT transition. Unlike the 2D XY model with a global U(1) symmetry where $T_{c 1}$ and $T_{c 2}$ coincide, in our case $T_{c 1}$ and $T_{c 2}$ do not coincide due to the presence of $\mathbb{Z}_{6}$ clock term in the free energy of Eq. (17). In the intermediate temperature $T_{c 1}<T<T_{c 2}$, we have an extended phase where both vortices and the clock anisotropy become irrelevant. The irrelevance of clock anisotropy indicates an emergent continuous U(1) symmetry that is shown in Fig. 5(b). Due to the emergent U(1) symmetry, the system behaves just like the low-temperature quasi-long-range ordered phase of the XY model without any anisotropy term and supports an algebraic spin correlation, and this thermal regime with $T_{c 1}<T<T_{c 2}$ is referred to as a BKT phase $[5,62,63]$. As long as the ground state is in the three-sublattice ordered phase, this BKT phase generically occurs in the finite temperature regime regardless of the parameters. For this reason, we plot the finite temperature phase diagram in Fig. 6 with a single choice of transverse field $h / J \approx 0.65$ that might be appropriate for $\mathrm{TmMgGaO}_{4}$ inside the three-sublattice ordered phase.

The underlying reason for the finite-temperature BKT physics in this context arises from the emergent U(1) symmetry. This emergent U(1) symmetry, however, no longer holds in the presence of external magnetic fields. The magnetic field breaks the time-reversal symmetry and brings about a $\mathbb{Z}_{3}$ clock anisotropy to the system [63],

$$
H_{3}=v_{3}\left(\psi^{3}+\psi^{* 3}\right),
$$

with $v_{3}$ linearly proportional to $B$. This $\mathbb{Z}_{3}$ clock term is always relevant at the phase transition. Therefore, the successive BKT transition scenario in thermal melting as well as an emergent continuous symmetry are no longer presented. Moreover, from Eqs. (17) and (18), we obtain the order parameter symmetry for each phase, as is shown in Fig. 7. We find that with magnetic field the order parameter symmetry 


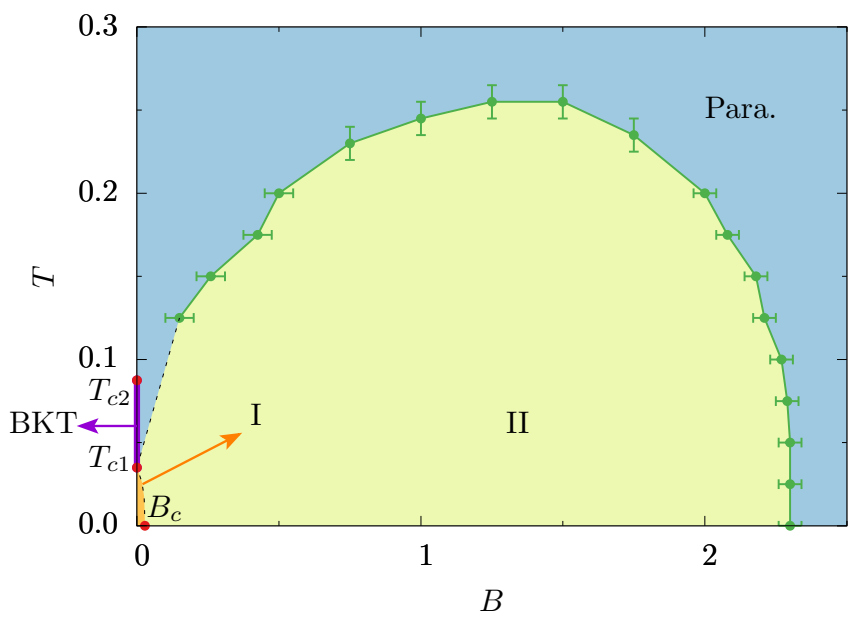

FIG. 6. Finite temperature phase diagram with an external magnetic field $B$ obtained from QMC simulations. The parameter we take is $J_{z z}=1, h=0.65$ where the ground state is a three-sublattice ordered state. The BKT phase at $B=0$ axis is marked by thick purple line. The lower and upper BKT transition points are $T_{c 2}=0.09(2) J_{z z}$ and $T_{c 1}=0.035(15) J_{z z}$ [56]. The green solid-dot line refers to firstorder transition while the upper and lower red dots at $B=0$ axis correspond to BKT and inverted BKT transitions, respectively. The phase boundaries (dash lines), when $B$ is very small, are difficult to capture in the current algorithm and are schematic here. The QMC simulation is performed with system sizes $L=6,12,24$ with the periodic boundary condition.

of the three-sublattice I is reduced from $\mathbb{Z}_{6}$ to $\mathbb{Z}_{3} \times \mathbb{Z}_{2}$. The symmetry is further reduced to $\mathbb{Z}_{3}$ in the intermediate threesublattice II state.

The finite-temperature phase diagram of the threesublattice state is shown in Fig. 6. According to Fig. 6, if one lowers the temperature from the trivial high-temperature paramagnetic phase at $B=0$, one experiences two successive transitions at $T_{c 2}$ and $T_{c 1}$. For BKT transitions, the correlation length diverges too fast near the thermal transition, the diverging behavior of the specific heat near the transition temperatures cannot be very well-observed experimentally or even numerically. This seems to be what happens for $\mathrm{TmMgGaO}_{4}$ : No diverging behavior is revealed in the specific heat data, instead only a tiny anomaly with a slightly broad peak is shown at $\sim 1 \mathrm{~K}[26,28]$.
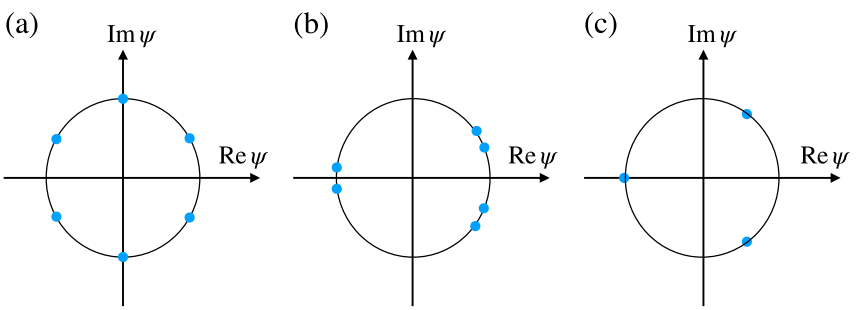

FIG. 7. Evolution of the order parameter manifold (marked as blue dots) with external magnetic field $B$. (a) The I state with $B=0$, (b) the I state with small $B \neq 0$, (c): the intermediate three-sublattice II state with larger $B$. The the symmetry of order parameter manifolds are $\mathbb{Z}_{6}, \mathbb{Z}_{3} \times \mathbb{Z}_{2}$ and $\mathbb{Z}_{3}$ for three cases, respectively.
With the magnetic field in Fig. 6, the $\mathbb{Z}_{3}$ clock anisotropy is introduced and the BKT scenario breaks down. For the intermediate II state that breaks the $\mathbb{Z}_{3}$ symmetry, there is only the first-order thermal transition. For the I state that breaks the $\mathbb{Z}_{2}$ symmetry in addition to $\mathbb{Z}_{3}$, the $\mathbb{Z}_{2}$ and $\mathbb{Z}_{3}$ symmetries should break at different temperatures, therefore one expects another Ising transition in addition to the first-order transition in the thermal melting. Unlike the BKT transitions that are weak and unclear in the heat capacity, these transitions are expected to show diverging signals (the Ising transition) or discontinuous signals (the first-order transition) in the thermodynamic measurements, as is shown in the magnetic specific heat data in Ref. [28]. However, for the transitions involving the I state where the magnetic field is weak, the divergent behavior is too weak to be observed experimentally or even numerically, as the system is close to the $B=0$ point where the BKT scenario happens. As it is at $T=T_{c 1}$ where the actual magnetic order appears, we expect that phase II starts from $T_{c 1}$ when the external field is applied. The dashed phase boundary in Fig. 6 is drawn schematically.

\section{Some experimental implications on BKT physics}

Experimentally, it is typically hard to detect BKT transitions in magnetic systems. Here we discuss how to determine BKT transition temperatures from experiments. Inside the BKT phase between $T_{c 2}$ and $T_{c 1}$, the algebraic spin correlation would lead to a quasi-Bragg peak at wave vector $K$. In principle, Bragg peaks may be distinguished from quasi-Bragg peaks by the elastic peak profile at the $K$ point, but this is again difficult. Further neutron-scattering studies might be useful to sort out the lower transition temperature $T_{c 1}$.

A relevant experimental prediction given by Damle in Ref. [63] is the singular uniform magnetic susceptibility along the $z$ direction in part of the BKT phase regime, despite absence of ferromagnetic order in this system. Due to the small energy scale of the interaction, the direct susceptibility measurements may not be able to give clear signals, especially because the impurity and disorder effects could affect very low-temperature thermodynamic behaviors. Somewhat equivalently, it is more convenient for us to examine the $S^{z}-S^{z}$ correlation at the $\Gamma$ point in the neutron-scattering measurement. From the available neutron data for $\mathrm{TmMgGaO}_{4}$ [26], we observe a clear upturn of the Bragg peak intensity at the $\Gamma$ point below $\sim 1 \mathrm{~K}$. The intensity is, however, 100 times smaller than the one at the $K$ point. It is known that, in the real materials, interlayer couplings could destroy the 2D BKT phase and convert the BKT phase transitions to a real ordering transition. We here tentatively identify $T_{c 2}$ as $\sim 1 \mathrm{~K}$, which is consistent with the $T_{c 2}$ obtained from the magnetic specific heat. From the phase diagram by Isakov and Moessner in Ref. [56] that indicated the phase boundary of the BKT phase, we conclude that $T_{c 1}$ is $\sim 0.5 \mathrm{~K}$. Thus, we postulate that the range of BKT phase is from $\sim 0.5 \mathrm{~K}$ to $\sim 1 \mathrm{~K}$ for $\mathrm{TmMgGaO}_{4}$.

Here we numerically examine the power-law behaviors of the $S^{z}-S^{z}$ correlation at $\Gamma$ point and at $K$ point inside the BKT phase. We have

$$
\chi_{\Gamma}=\frac{L^{2}}{\beta}\left\langle\left|\int_{0}^{\beta} d \tau m_{\Gamma}(\tau)\right|^{2}\right\rangle
$$




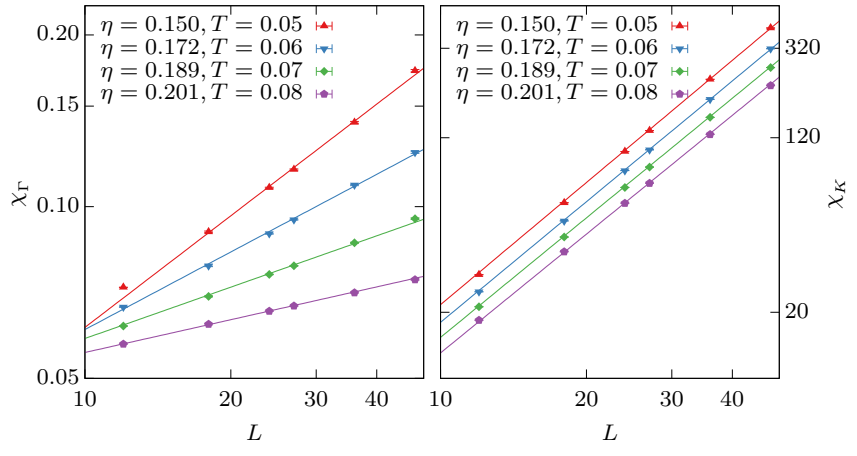

FIG. 8. Susceptibilities $\chi_{\Gamma}$ and $\chi_{K}$ versus the linear system size $L$ for four different temperatures when $h=0.65$ in BKT phase. Solid lines are power-law forms $\sim L^{2-9 \eta}$ (on the left panel) and $\sim L^{2-\eta}$ (on the right panel). Small systems maybe will deviate from power-law forms due to the effect of large-finite size scaling.

and the order parameter susceptibility

$$
\chi_{K}=\frac{L^{2}}{\beta}\left\langle\left|\int_{0}^{\beta} d \tau m_{K}(\tau)\right|^{2}\right\rangle,
$$

where $m_{\mathbf{q}} \equiv \frac{1}{N} \sum_{i} S_{i}^{z} \exp \left(i \mathbf{q} \cdot \mathbf{r}_{i}\right)$ with $N$ the system size. According to the previous field theoretical analysis by Damle [63], they are expected to scale with the system size $L$ as

$$
\begin{aligned}
& \chi_{\Gamma} \sim L^{2-9 \eta(T)}, \\
& \chi_{K} \sim L^{2-\eta(T)},
\end{aligned}
$$

with $1 / 9 \leqslant \eta(T) \leqslant 2 / 9$ in part of the BKT phase [63]. The QMC results are shown in Fig. 8 and both fit rather well to Eqs. (21) and (22). Although there is always no ferromagnetic long-range order in the BKT phase, $\chi_{\Gamma}$ is still divergent with the system size in part of the phase region.

\section{DYNAMIC PROPERTIES FROM ORTHOGONAL OPERATOR APPROACH AND SELECTIVE MEASUREMENTS}

The previous section deals with the phase diagram and the magnetic ordering structures. These properties are static magnetic properties. To provide more information about the system, we here explore the dynamic properties from the orthogonal operator approach and the selective measurements. In Sec. V A, we explain the orthogonal operator approach. In Sec. VB, we turn to the selective measurement that directly applies the orthogonal operator approach for Tm-based triangular lattice antiferromagnets.

\section{A. Orthogonal operator approach}

Even though the in-plane components are nonzero, they are not visible from the experiments. These hidden-order-like features can be revealed from an approach called orthogonal operator approach $[30,35]$. The notion of hidden order was introduced into condensed-matter physics in the study of the compound $\mathrm{URu}_{2} \mathrm{Si}_{2}$ [64]. The order parameter associated with the hidden order does not couple strongly with the conventional experimental probe such that the order does not explicitly show up in the usual experimental probes. To identify the nature of the hidden order, our simple suggestion was to find the physical observables whose operators do not commute with the proposed hidden order operators, and at the same time make sure these observables are ready to detect experimentally. These operators are referred to as orthogonal operators. The dynamic correlations or spectra of these operators would reveal the structure and the nature of the underlying hidden orders. These thoughts have been explored for the quadrupolar orders and the octupolar orders of triangular lattice magnets $[30,35]$ as well as the spin nematics in frustrated magnets [65].

Because the nonvanishing in-plane components are induced by the intrinsic transverse field, strictly speaking, they are not the Landau symmetry-breaking orders. Nevertheless, their presence and behavior are very much similar to the roles of the hidden orders and thus can be understood in a similar manner.

\section{B. Selective measurements}

Having figured out the phase diagrams in the previous section, we here explain the experimental consequences for the dynamics from the selective measurements and the orthogonal operator approach. The three-sublattice order that we found from the model is characterized by the order parameter $\psi$ defined by the dipolar magnetization, which is directly reflected as the magnetic Bragg peaks at the $K$ point. Meanwhile, due to the intrinsic crystal field, there is always a nonvanishing expectation value in the transverse components that arises not from the spontaneous symmetry breaking but from the intrinsic polarization effect. Since the transverse components are the magnetic multipoles, they do not directly couple to the neutron spins and hence are hidden in the neutron probes. Due to the peculiar local moment structure of this system, however, the elementary excitations of the multipole moment can be measured in the dynamic probes such as the inelastic neutron scattering, owing to the noncommutative relation between the dipole and the multipole moments. This specific idea was initially pointed out in the context of the non-Kramers doublets [30] and also applies here. As the neutron spins only directly couple to the dipole components, in the inelastic neutron scattering what is measured is the $S^{z}-S^{z}$ correlation,

$$
\mathcal{S}^{z z}(\boldsymbol{q}, \omega)=\frac{1}{2 \pi N} \sum_{i j} \int_{-\infty}^{+\infty} \mathrm{d} t e^{i \boldsymbol{q} \cdot\left(\mathbf{r}_{i}-\mathbf{r}_{j}\right)-i \omega t}\left\langle S_{i}^{z}(0) S_{j}^{z}(t)\right\rangle,
$$

and the transverse component correlation is not directly visible in the neutron-scattering measurement. Based on the above selective measurement, a regular neutron-scattering measurement would behave like a polarized neutron measurement that automatically selects the $S^{z}-S^{z}$ correlation. As the neutron spin detects the longitudinal dipole moments, it "flips" the multipole moment that is orthogonal to the dipole moment, creating the coherent spin-wave excitations. Therefore, in an inelastic neutron scattering experiment, what is measured is the elementary excitation of the multipole components that contains the information on the underlying hidden multipole structures. We have calculated the dynamic structure factors for three representative parameters. The results are 

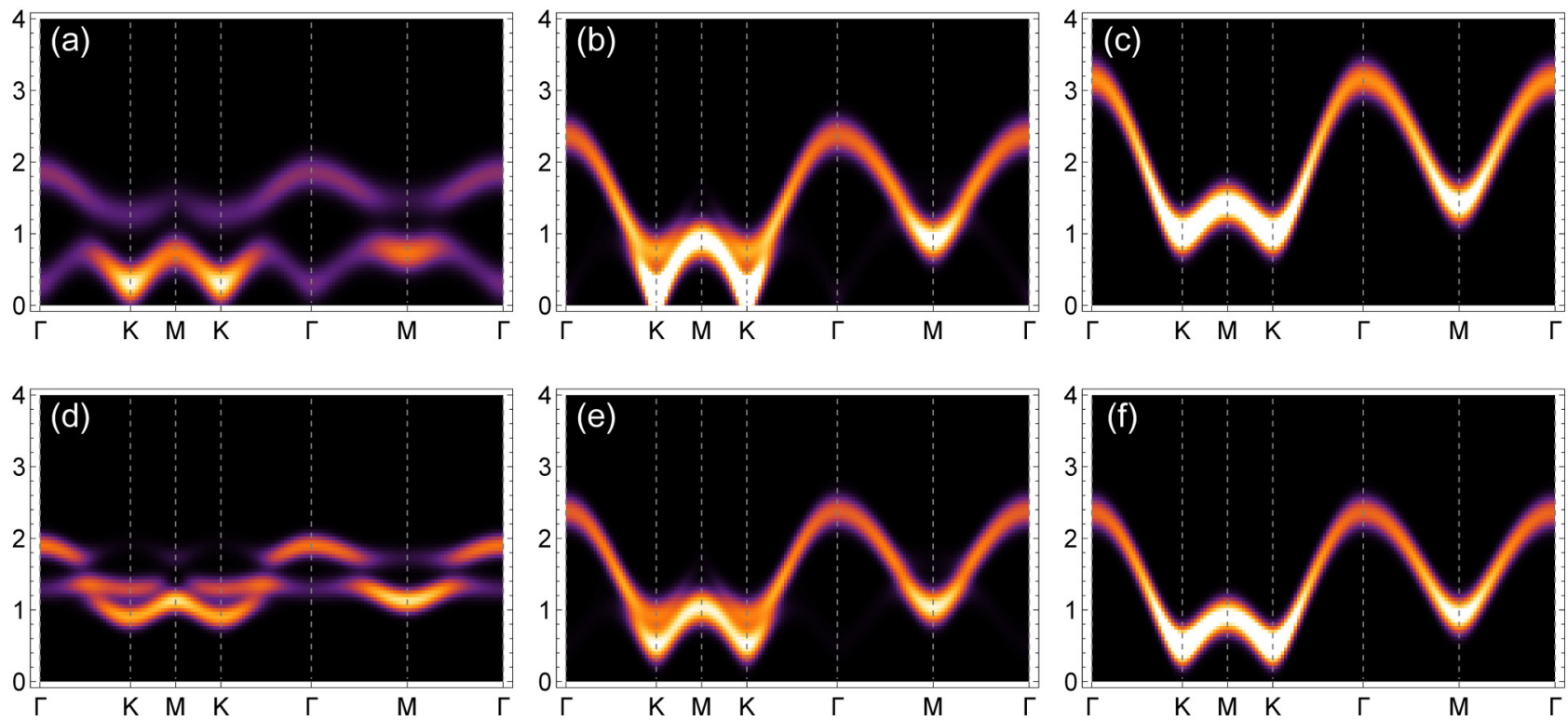

FIG. 9. Dynamical correlation function $\mathcal{S}^{z z}(\boldsymbol{q}, \omega)$ calculated within the linear spin-wave theory of (a), (b) the three-sublattice I state, (d), (e) the intermediate three-sublattice II state, and (c), (f) the paramagnetic (or polarized) state. The parameter we take for the representative points are (a) $h=0.8, B=0$; (b) $h=1.3, B=0$; (c) $h=2, B=0$, (d) $h=0.8, B=1.3$; (e): $h=1.3, B=1.3$; (f) $h=1.3, B=1.8$. In all cases, we take $J_{z z}=1$.

shown in Fig. 9. For the paramagnetic (or Ising disordered) side, there is only one branch of excitation, reflecting the uniform structure of the paramagnetic (or Ising-disordered) phase with a ferro-multipole order $\left\langle S^{y}\right\rangle$ [see Fig. 9(c)]. If another Tm-based triangular lattice material is located in this parameter regime and phase, there will be no transition through all temperatures but the excitation spectrum surprisingly becomes more coherent as the temperature is lowered, despite the absence of any ordering. This phenomenon can be quite striking from the experimental perspective.

Meanwhile, for the three-sublattice ordered state, one can roughly identify two branches of excitations in Fig. 9(b) and clearly identify two branches of excitations in Fig. 9(a). The experimental situation [26] in $\mathrm{TmMgGaO}_{4}$ is more close to Fig. 9(b) that shows a reasonable agreement with the experimental data. In Fig. 9(b), we choose the specific parameter $h / h_{c}^{\mathrm{MF}} \simeq 0.87$ where $h_{c}^{\mathrm{MF}}$ is the critical field of the mean-field theory. The counting of the branch number immediately brings up a question that the number of branches in the experiment is inconsistent with the number of magnetic sublattices. This question was not raised in Ref. [26] and is addressed here. In fact, our honest linear spin-wave calculation of the full spectra in the Appendix gives three branches of dispersions that correspond to the three-sublattice magnetic structure. The reason that the $S^{z}-S^{z}$ correlation looks like two branches is because the selective measurement makes the intensity of part of the excitation spectra rather weak such that the spectra look like two branches. This indicates the incompleteness of the selective measurements. Other dynamic measurements such as optics and $\mathrm{THz}$ may avoid the selective measurement issue and provide complementary information about the excitations here.

For the specific $\mathrm{TmMgGaO}_{4}$ material, the previous neutron-scattering experiment shows a tiny spin gap at the $K$ point [26]. This is expected as the model and the system do not have any continuous symmetry to support any gapless Goldstone mode. The reason that the gap is tiny is a common consequence of the quantum order by disorder [66] for the TFIM with the antiferromagnetic Ising interaction on the triangular lattice. This tiny-gapped mode is sometimes referred to as a pseudo-Goldstone mode, as it appears as a breaking of continuous symmetry at the quadratic or linear spin-wave theory level [66].

\section{MORE EFFECTS FROM EXTERNAL MAGNETIC FIELD}

The external magnetic field not only enriches the phase diagram but also generates more experimental consequences to be examined. In this section, we will first focus on the static properties of the system such as the magnetization and static spin structure factor under the external magnetic field, and then explore the dynamic properties of the system.

\section{A. Susceptibility, magnetization, and non-monotonic ordering}

Except in a small parameter regime near the critical point (see Fig. 3) where the magnetic field could drive a re-entrant transition by crossing the three-sublattice ordered one, we do not expect phase transitions upon increasing temperatures with or without the external field for the paramagnetic (or the Ising disordered) state that preserves all the lattice symmetries. This behavior is fundamentally different from those of the three-sublattice ordered state, which can be used to identify these two phases without performing the neutron scattering experiments. The magnetic susceptibility $\chi^{z z}$ as a function of the temperature is calculated via QMC and is plotted in Fig. 10(a). At high temperatures, the magnetic susceptibility satisfies the Curie-Weiss law with 

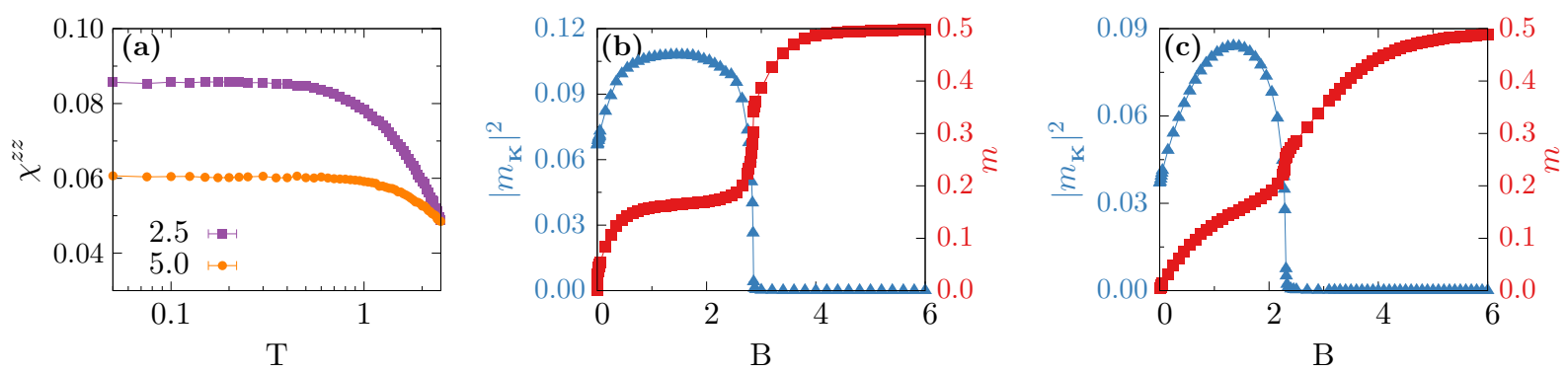

FIG. 10. (a) The magnetic susceptibility $\chi^{z z}$ versus the temperature $T$ for the paramagnetic (or the Ising disordered) state. Here we take the parameter $J_{z z}=1, h=2.5,5.0$. The magnetic susceptibility is defined as $\chi^{z z}=\partial m / \partial B$, where $m=\left(\sum_{i} S_{i}^{z}\right) / N$ is the dipolar magnetization per site. (b), (c) Magnetization $m$ (in red) and the magnetic Bragg peak $\left|m_{K}\right|^{2}$ (in blue) for the three-sublattice state at low temperatures. The parameter we take is $J_{z z}=1$; for (b) $h=0.25$, for (c) $h=0.65$. All results are calculated through QMC with the system size $L=12$ and $\beta=80$. The error bars are much smaller than the points.

$\chi^{z z} \simeq C /\left(T-\Theta_{\mathrm{CW}}\right)$, where $C$ is the Curie constant and $\Theta_{\mathrm{CW}}=-3 J_{z z} / 2$ where one can extract the exchange parameter $J_{z z}$. There is a crossover to the low temperature behavior where $\chi^{z z}$ saturates to a constant. This is because the Hamiltonian does not have any continuous symmetry and the total magnetization is not a good quantum number to label the many-body states. Within a simple mean-field theory, we find the low-temperature $\chi^{z z}$ is given as

$$
\left.\chi^{z z}\right|_{T \rightarrow 0} \approx \frac{1}{6 J_{z z}+2 h} .
$$

Compared to the QMC data, we find that at large $h$, they coincide very well. Therefore, one can extract the model parameter $J_{z z}$ and $h$ simply from the high-temperature and low-temperature behaviors of $\chi^{z z}$ if the system is located in the paramagnetic phase. The above relation is especially useful if the ground state of the system is in the Ising disordered phase.

We continue to discuss the magnetization process of the three-sublattice state that can be relevant for the specific material $\mathrm{TmMgGaO}_{4}$. In the absence of the external magnetic field, the three-sublattice ordering arises from the quantum orderby-disorder mechanism. The spin excitation gap is relatively small [see Figs. 9(a) and 9(b)]. This property makes the threesublattice state fragile against the external magnetic field. A small external field at $B_{c 1}$ will cause the closing of the spin gap and drive the system towards the intermediate quasiplateau state. Here quasiplateau is used as the total magnetization is not conserved. With the increasing external magnetic field, the spin gap reopens and the intermediate state becomes stable. Further increasing magnetic field, the spin gap drops until the system is driven to a polarized state by the magnetic field at $B_{c 2}$ above which the system is smoothly connected to the fully polarized one.

The presence of the intermediate quasiplateau state renders the the magnetization process nontrivial, as shown in Fig. 10 from the QMC calculation. For small $h$, the magnetization curve shows a clear $1 / 3$ quasiplateau feature in the intermediate regime. Meanwhile, deep in the quasiplateau state, the system has an approximate 2-up-1-down structure that contributes to a robust three-sublattice ordering compared to the case without the external field. Therefore, in the elastic neutron-scattering experiments, the intensity of the magnetic Bragg peak $\left|m_{K}\right|^{2}$ (proportional to $|\psi|^{2}$ ) is expected to show nonmonotonic behaviors: deep in the quasiplateau state the intensity is large, while approaching the three-sublattice state I the intensity is expected to decrease; the intensity is also expected to decrease when the field is large enough where the system becomes nearly polarized [see Fig. 10(b)].

For the case relevant with $\mathrm{TmMgGaO}_{4}$ where the transverse field $h$ is comparable to the exchange $J_{z z}$, in the quasiplateau regime, the 2-up-1-down structure is heavily distorted, therefore the quasiplateau feature of the intermediate regime is not clearly observed in the magnetization curve. Instead, the line shape curves slightly downward at $B_{c 2}$ [see Fig. 10(c)]. This feature is found in the magnetization data of $\mathrm{TmMgGaO}_{4}$ at about $2.5 \mathrm{~T}$, which marks the transition field $B_{c 2}$ [26-29]. Above $B_{c 2}$ the system becomes polarized, but not fully aligned along the $z$ direction due to the presence of the intrinsic transverse field. To allow the magnetization to approach the saturation value, a larger external field has to be applied. This feature is in stark contrast to ordinary systems where the internal transverse field is absent. For the magnetic Bragg peak $\left|m_{K}\right|^{2}$, the nonmonotonic behavior persists with large transverse fields [see Fig. 10(c)].

\section{B. Dynamic properties in magnetic fields}

Here we discuss the dynamical properties in the presence of external magnetic field. With applying small external magnetic field, the gap first decreases and closes at $B_{c 1}$. As $B_{c 1}$ is typically small, this phenomenon is subtle and can be hard to observe experimentally. With increasing magnetic field, the system gap reopens across $B_{c 1}$ as it enters the intermediate II regime. In the II regime, the gap behaves nonmonotonically: the gap first increases, reaches maximum and drops until the system becomes polarized at $B_{c 2}$ via a first-order transition. As this transition being first-order, the gap does not close across $B_{c 2}$.

We have calculated the spin excitation spectra with magnetic field, as shown in Figs. 9(d)-9(f). From Fig. 9(d), we can clearly see three spin-wave branches, consistent with the three-sublattice magnetic order. Therefore, the selection rule breaks down with magnetic field. Another observation is that there remains nonzero intensity even in the fully polarized state, see Fig. 9(f). This is a peculiar feature for our nondegenerate dipole-multipole doublet systems due to the intrinsic transverse field: The spins are tilted to acquire nonzero 
transverse components, which results in the non-vanishing intensity in the polarized state.

\section{DISCUSSION}

\section{A. Summary for $\mathrm{TmMgGaO}_{4}$}

In this paper, we have performed a theoretical study on the triangular lattice TFIM relevant with the $\mathrm{TmMgGaO}_{4}$ material. We clarify the intrinsic origin of transverse field of this material as the crystal-field splitting. We established the full phase diagram by combining the mean-field theory and the QMC simulation. We discuss the continuous symmetry and BKT physics that emerge in the thermal melting and at the quantum critical point. We explain the properties of phases in the neutron-scattering measurement and the thermodynamic experiments. The available experimental data show that this material at zero field is well consistent with the TFIM with the three-sublattice intertwined dipolar and multipolar ordered ground state.

We mention a couple recent works on $\mathrm{TmMgGaO}_{4}$ and the TFIM on triangular lattice. A recent numerical-oriented work [67] explored our proposed effective model for $\mathrm{TmMgGaO}_{4}$ using more updated numerical techniques and focused on the numerical aspects of the model. Their results supported the validity of the TFIM for $\mathrm{TmMgGaO}_{4}$. Reference [67] suggested the system first enters the BKT phase at $\sim 4 \mathrm{~K}$ and then enters the three-sublattice ordered state at $\sim 1 \mathrm{~K}$. This differs from the results of the current work, where we have $\sim 1 \mathrm{~K}$ for the upper BKT transition and $\sim 0.5 \mathrm{~K}$ for the lower one. They further established the roton mode at the $M$ point inside the three-sublattice ordered state. This is probably due to the presence of the second-neighbor interaction. One may understand this in analogous with the supersolidity and the roton mode in the spin-1/2 XXZ model or repulsive hard-core boson model at half-filling on the triangular lattice where the roton condensation leads to the $S^{z}$ order $[68,69]$ on top of the transverse component order. The difference is that the TFIM here develops an emergent U(1) symmetry and cannot have supersolidity while the XXZ model has a global $\mathrm{U}(1)$ symmetry. One may further consider the effect of the dipole-dipole interaction and other effects on this roton mode.

Another quite recent experimental work [29] supplemented the early thermodynamic results [28] with neutron-diffraction measurements and corrected the early claim of a pure Ising model with more analysis. In the recent work [29], the authors added the transverse field and suggested the $\mathrm{Mg} / \mathrm{Ga}$ site disorder could create a (random) distribution of the transverse field. They argued that this site disorder could be the origin of their proposed partial up-up-down order. Based on the neutron-diffraction and thermodynamic measurements, Ref. [29] compared the parameters of different couplings of the model with finite-size exact diagonalization calculation and supported the proposal of a TFIM with random disorders. Since Ref. [29] raised the possible issue of random disorders, we agree that the quantitative behaviors of the thermodynamic results might be more sensitive to disorder effects on the exchange and $g$ factors as well as the residual coupling to the high-order multipolar moments. On the other hand, the random exchange and/or the random transverse field would lead to the line broadening with a similar range of energies in the spin-wave spectrum [29]. Although a well-defined spin-wave spectrum with a clear dispersion was recorded in the inelastic neutron-scattering measurement and reported in Ref. [26], we still think more data-rich experiments are needed at this stage if one hopes to extract more quantitive information. For example, one could carry out the inelastic neutron-scattering measurements as a function of the external magnetic field and establish the excitation spectrum in the (more robust) three-sublattice ordered state of the phase II. One can combine the results of zero field and finite fields and give an estimate of the transverse field distribution from the linewidth of the excitations after subtracting the broadening due to the magnon interactions and the instrument resolution.

In our analysis here, we did not consider the random disorder effect that was actually raised after the first online version of the current work in Ref. [28], and were unable to provide or address much more detailed numerical and quantitative aspects that relate to the experiments quantitatively. We focus more on the generic and qualitative physics that may be more robust in the experiments. Regardless of the specific material, it will be interesting to understand the fate of the finite-temperature BKT phase in the presence of quenched random disorders, and this may be analyzed with the perturbative renormalization calculation within the BKT phase.

\section{B. Connection to upper branch magnetism}

In fact, the magnetism of $\mathrm{TmMgGaO}_{4}$ belongs to the category of systems with upper branch magnetism. The notion of upper branch magnetism was introduced in Ref. [70]. It refers to the case where the local crystal-field environment simply favors a nonmagnetic state while the superexchange interaction prefers magnetic states of some sort. For the specific illustrative example in Ref. [70], the local crystal-field ground state is a singlet and the first excited states are a twofold degenerate doublet. The specific system there was modeled as an effective spin-1 magnet and the crystal-field splitting was modeled as a single-ion anisotropy for the spin-1 moment.

In what sense is $\mathrm{TmMgGaO}_{4}$ regarded as upper branch magnetism? The magnetism cannot occur if there is no exchange interaction between the Tm local moments. Crudely speaking, it is the exchange interaction that "drag down" the excited energy level. More precisely, it is the nontrivial quantum mechanical interplay between the intrinsic transverse field and the Ising exchange that gives rise to the magnetism and the associated coherent excitation. What do we expect experimentally if the system is controlled more by the singleion physics? The magnetism will be gone. Despite that, the coherent magnetic excitation would persist. This may occur in some other systems.

\section{Extension to other Tm-based compounds}

A series of rare-earth triangular lattice magnets has been summarized in Ref. [30]. We expect that other materials, especially some Tm-based compounds, can also be described by the TFIM and share similar physics with $\mathrm{TmMgGaO}_{4}$. The Tm-based magnetism is not a common subject in quantum magnetism of rare-earth systems. Some of the insights that 
we learn from $\mathrm{TmMgGaO}_{4}$ could be applied to other $\mathrm{Tm}$ magnets. In the following, we survey the existing Tm magnets and explain the physics in them.

\section{Tm spinels and Tm pyrochlores}

The Tm spinel, $\mathrm{MgTm}_{2} \mathrm{Se}_{4}$, has been studied by the neutron-scattering measurement [71]. The crystal electric field states were carefully studied in Ref. [71]. It turns out that the crystal-field ground state and the first excited state are similar to the ones in $\mathrm{TmMgGaO}_{4}$. They are separated from other excited levels by an energy gap more than $10 \mathrm{meV}$. The wave functions of the lowest two states are

$$
\begin{aligned}
\left|\Psi_{g}\right\rangle= & 0.66960|6\rangle+0.14821|3\rangle+0.24361|0\rangle \\
- & 0.14821|-3\rangle+0.66960|-6\rangle, \\
\left|\Psi_{e}\right\rangle= & -0.70097|6\rangle-0.092966|3\rangle \\
& \quad-0.092966|-3\rangle+0.70097|-6\rangle,
\end{aligned}
$$

and the energy separation between them is about $0.885 \mathrm{meV}$. Similar to $\mathrm{TmMgGaO}$, one could introduce an effective spin$1 / 2$ degree of freedom that operates on these two states.

The Tm-based pyrochlore has rarely been studied. The crystal-field levels of $\mathrm{Tm}_{2} \mathrm{Ti}_{2} \mathrm{O}_{7}$ were computed in Ref. [72]. It was found that the crystal-field ground state is a singlet with the wave function

$\left|\Psi_{g}\right\rangle=0.147|6\rangle-0.692|3\rangle-0.692|-3\rangle-0.147|-6\rangle$,

and the first excited state is a doublet with twofold degeneracy. This crystal-field-level setting is identical to the specific case that was considered in Ref. [70]. The energy separation between the ground-state singlet and the first excited doublet is of the order of $10 \mathrm{meV}$, so it is not in the weak crystal-field regime for rare-earth magnets. It is likely that other isostructural Tm-based pyrochlores could have a smaller crystal-field gap and allow more interesting magnetism to happen.

\section{Tm honeycomb lattice and Tm Kagomé lattice magnets}

Here we extend some of our thoughts to other 2D systems. We start with the honeycomb magnet $R \mathrm{Ni}_{3} \mathrm{Al}_{9}$ where $R$ is the rare-earth ion and Tm is a member of them [73]. These materials have both conduction electrons and local moments, so it is a conductor and there is a Kondo physics in some of them. The local moment magnetism is from the rare-earth moments. We focus the discussion on the Tm-based materials. Other materials in this family such as the Yb-based ones could involve Kitaev and other anisotropic spin interactions between the local moments and are also worth further investigation. The magnetization measurement in the single crystal sample of $\mathrm{TmNi}_{3} \mathrm{Al}_{9}$ is quite similar to the one in $\mathrm{TmMgGaO}_{4}$, where the out-of-plane response is dominant and the in-plane response is negligible. There are two possibilities for the Tm magnetism in $\mathrm{TmNi}_{3} \mathrm{Al}_{9}$. The first possibility is that the $\mathrm{Tm}$ local moment is a (degenerate) non-Kramers doublet. The other possibility is that the Tm local moment is a nondegenerate $\mathrm{DM}$ doublet like the one in $\mathrm{TmMgGaO}_{4}$, and the effective model for the Tm magnetism would be a TFIM. Due to the presence of the itinerant electrons, the Ising interaction may involve further neighbors. This material develops a mag- netic order at $2.9 \mathrm{~K}$ from the thermodynamic and transport measurements. From the experience about $\mathrm{TmMgGaO}_{4}$, we expect a coherent excitation spectrum. This may be confirmed by further experiments with neutron scattering measurements.

Tm-based Kagomé magnets have been explored recently [74-76], and effective spin-1/2 degrees of freedom are used to describe the $\mathrm{Tm}$ magnetism. Unlike the triangular lattice and the honeycomb lattice, the point-group symmetry does not involve an on-site threefold rotation, and there is no nonKramers doublet on the Kagomé lattice. Thus there is always an intrinsic splitting between the two relevant crystal-field levels of the $\mathrm{Tm}^{3+}$ ion. Because the $\mathrm{Tm}^{3+}$ singlets are not the same kind of singlets as the ones in $\mathrm{TmMgGaO}_{4}$, the exchange part of the interaction is not simply by the Ising model.

\section{Tm double perovskites}

Another class of Tm magnets is the Tm-based double perovskite. Unlike the rare-earth pyrochlores and the rare-earth triangular lattice magnets, these materials have not been well studied before. Here Tm ions form a FCC lattice. Only two Tm-based double perovskites $\mathrm{Ba}_{2} \mathrm{TmSbO}_{6}$ and $\mathrm{Ba}_{2} \mathrm{TmBiO}_{6}$ have been studied [77]. Besides the basic thermodynamic and structural measurements at high temperatures, very little information is known for these two materials. Thus, we cannot extract much more physical understanding for the time being. But these two materials remain as good candidates for frustrated FCC systems with spin-orbit-entangled local moments [78].

\section{General expectation for intrinsic quantum Ising magnets}

From our study of $\mathrm{TmMgGaO}_{4}$ and the discussion on many other Tm-based magnets, we think the intrinsic quantum Ising magnets can widely exist in nature. The $\mathrm{Tm}^{3+}$ ion in the $\mathrm{D}_{3 d}$ crystal field is a bit special. Even though the $\mathrm{D}_{3 d}$ point group is a high-symmetry group with many operations, the singlet representations are allowed here and correspond to the two low-lying singlets in $\mathrm{TmMgGaO}_{4}$. In more general cases [17], we do not have such a high-symmetry point group, and thus we think the intrinsic transverse field can be more common in rare-earth magnets with lower crystal-field symmetries. Nevertheless, a lower crystal-field symmetry is not a sufficient condition to make the two low-lying singlet closer in energies. Instead, one could imagine the lower crystal-field symmetry is obtained by weakly breaking a higher crystal-field symmetry. An example is the tripod Kagomé magnet that can be obtained from the pyrochlore magnets by replacing the apical rare-earth atoms with other nonmagnetic ones such that the originally degenerate non-Kramers doublet picks up a small energy splitting. For the half-integer spin local moments, the Kramers theorem necessarily demands a twofold degeneracy that makes the quantum Ising model description impossible. Thus, an interesting direction to search for the intrinsic quantum Ising magnets is to examine the rare-earth magnets with low crystal-field symmetries and integer-spin local moments.

\section{ACKNOWLEDGMENTS}

C.-J.H. thanks Prof. Youjin Deng for useful discussions on QMC algorithm and providing computational 
resources supported by the National Natural Science Foundation of China under Grant No. 11625522. We also thank Kedar Damle for a conversation. This work is further supported by the Ministry of Science and Technology of China with Grants No. 2018YFGH000095, No. 2016YFA0301001, No. 2016YFA0300500, and by Shanghai Municipal Science and Technology Major Project with Grant No. 2019SHZDZX01, and by the Research Grants Council of Hong Kong with General Research Fund Grant No. 17303819.

\section{APPENDIX: RESULTS FROM LINEAR SPIN-WAVE THEORY}

In this Appendix, we provide the linear spin-wave theory and results for the magnetic excitations in the three-sublattice magnetic orders. The reason that we do this calculation is to clarify the discrepancy between the number of the magnetic sublattices and the numbers of the measured magnon branches.

For the three-sublattice magnetic ordered states, the system has $\sqrt{3} \times \sqrt{3}$ magnetic unit cell; each spin can be labeled by combination of magnetic unit cell position $\mathbf{r}$ and sublattice index $s(s=1,2,3)$. The mean-field ground states can be obtained by Weiss mean-field theory, where the mean-field spin orientations for each sublattice $s$ can be labeled by unit vector $\mathbf{n}_{s}$. Then one can always associate two unit vectors $\mathbf{u}_{s} \cdot \mathbf{n}_{s}=0$ and $\mathbf{v}_{s}=\mathbf{n}_{s} \times \mathbf{u}_{s}$ so $\mathbf{n}_{s}, \mathbf{u}_{s}$ and $\mathbf{v}_{s}$ are orthogonal with each other. Next we perform Holstein-Primakoff transformation for the spin operator $\mathbf{S}_{\mathbf{r} s}$,

$$
\begin{gathered}
\mathbf{n}_{s} \cdot \mathbf{S}_{\mathbf{r} s}=S-b_{\mathbf{r} s}^{\dagger} b_{\mathbf{r} s}, \\
\left(\mathbf{u}_{s}+i \mathbf{v}_{s}\right) \cdot \mathbf{S}_{\mathbf{r} s}=\left(2 S-b_{\mathbf{r} s}^{\dagger} b_{\mathbf{r} s}\right)^{\frac{1}{2}} b_{\mathbf{r} s}, \\
\left(\mathbf{u}_{s}-i \mathbf{v}_{s}\right) \cdot \mathbf{S}_{\mathbf{r} s}=b_{\mathbf{r} s}^{\dagger}\left(2 S-b_{\mathbf{r} s}^{\dagger} b_{\mathbf{r} s}\right)^{\frac{1}{2}} .
\end{gathered}
$$

After performing the Fourier transformation,

$$
b_{\mathbf{r} s}=\sqrt{\frac{3}{N}} \sum_{\mathbf{k} \in \overline{\mathrm{BZ}}} b_{\mathbf{k} s} e^{i \mathbf{R}_{\mathbf{r} s} \cdot \mathbf{k}},
$$

the spin Hamiltonian can be rewritten in terms of boson bilinears as

$$
H_{\mathrm{sw}}=\frac{1}{2} \sum_{\mathbf{k} \in \overline{\mathrm{BZ}}} \Psi(\mathbf{k})^{\dagger} h(\mathbf{k}) \Psi(\mathbf{k})+\text { const }
$$

where

$$
\Psi(\mathbf{k})=\left[b_{\mathbf{k} 1}, b_{\mathbf{k} 2}, b_{\mathbf{k} 3}, b_{-\mathbf{k} 1}^{\dagger}, b_{-\mathbf{k} 2}^{\dagger}, b_{-\mathbf{k} 3}^{\dagger}\right]^{T},
$$

$h(\mathbf{k})$ is a $6 \times 6$ Hermitian matrix, and $\overline{\mathrm{BZ}}$ is the magnetic Brillouin zone. Then we can Bogoliubov diagonalize $H_{\text {sw }}$ with $\Psi(\mathbf{k})=T_{\mathbf{k}} \Phi(\mathbf{k})$, where

$$
\Phi(\mathbf{k})=\left[\beta_{\mathbf{k} 1}, \beta_{\mathbf{k} 2}, \beta_{\mathbf{k} 3}, \beta_{-\mathbf{k} 1}^{\dagger}, \beta_{-\mathbf{k} 2}^{\dagger}, \beta_{-\mathbf{k} 3}^{\dagger}\right]^{T},
$$

is the diagonalized basis and $T_{\mathbf{k}}$ is the transformation matrix. Details of the diagonalization can be referred to in Refs. [79-81]. The diagonalized Hamiltonian reads

$$
\begin{aligned}
H_{\mathrm{sw}} & =\frac{1}{2} \sum_{\mathbf{k} \in \overline{\mathrm{BZ}}} \Phi(\mathbf{k})^{\dagger} E(\mathbf{k}) \Phi(\mathbf{k})+\text { const } \\
& =\sum_{\mathbf{k} \in \overline{\mathrm{BZ}}} \omega_{\mathbf{k} s} \beta_{\mathbf{k} s}^{\dagger} \beta_{\mathbf{k} s}+\text { const }
\end{aligned}
$$

where $E(\mathbf{k})=\operatorname{diag}\left[\omega_{\mathbf{k} 1}, \omega_{\mathbf{k} 2}, \omega_{\mathbf{k} 3}, \omega_{-\mathbf{k} 1}, \omega_{-\mathbf{k} 2}, \omega_{-\mathbf{k} 3}\right]$. Within this formalism, we find that the coherent contribution to the $\mathcal{S}^{z z}$ correlator takes the following form:

$$
\mathcal{S}^{z z}(\mathbf{k}, \omega)=\frac{S}{6} \sum_{s=1}^{3}\left[T_{\mathbf{k}}^{\dagger} \mathbf{U}^{z}\left(\mathbf{U}^{\mathbf{z}}\right)^{\dagger} T_{\mathbf{k}}\right]_{s+3, s+3} \delta\left(\omega-\omega_{-\mathbf{k} s}\right),
$$

where $\mathbf{U}^{z}$ is a $6 \mathrm{D}$ vector

$$
\begin{aligned}
\mathbf{U}^{z}= & {\left[u_{1}^{z}+i v_{1}^{z}, u_{2}^{z}+i v_{2}^{z}, u_{3}^{z}+i v_{3}^{z},\right.} \\
& \left.u_{1}^{z}-i v_{1}^{z}, u_{2}^{z}-i v_{2}^{z}, u_{3}^{z}-i v_{3}^{z}\right]^{T} .
\end{aligned}
$$

Due to the quantum fluctuation, the magnetic orders are suppressed from the mean-field values, as a result, the bandwidth of the single-magnon spectra will be renormalized. This is a well-known feature of the linear spin-wave theory [82]. If one is interested in more quantitative features, one could use a more involved renormalized spin-wave theory that takes into account the suppression of the magnetic orders by quantum fluctuations [82]. However, the linear spin-wave theory does provide a useful understanding of the structure of the magnetic excitations. In our spin-wave calculation, there are three branches of dispersions that are consistent with the number of the magnetic sublattices.
[1] G. Wannier, Phys. Rev. 79, 357 (1950).

[2] K. Kanô and S. Naya, Prog. Theor. Exp. Phys. 10, 158 (1953).

[3] M. J. Harris, S. T. Bramwell, D. F. McMorrow, T. H. Zeiske, and K. W. Godfrey, Phys. Rev. Lett. 79, 2554 (1997).

[4] A. P. Ramirez, A. Hayashi, R. J. Cava, R. Siddharthan, and B. Shastry, Nature 399, 333 (1999).

[5] R. Moessner, S. L. Sondhi, and P. Chandra, Phys. Rev. Lett. 84, 4457 (2000).

[6] R. Moessner and S. L. Sondhi, Phys. Rev. B 63, 224401 (2001).

[7] M. Hermele, M. P. A. Fisher, and L. Balents, Phys. Rev. B 69, 064404 (2004).
[8] J. Röchner, L. Balents, and K. P. Schmidt, Phys. Rev. B 94, 201111(R) (2016).

[9] P. Nikolić and T. Senthil, Phys. Rev. B 71, 024401 (2005).

[10] J. Villain, R. Bidaux, J.-P. Carton, and R. Conte, J. Phys. France 41, 1263 (1980).

[11] E. F. Shender, ZhETF 83, 326 (1982) [JETP 56, 178 (1982)].

[12] M. Powalski, K. Coester, R. Moessner, and K. P. Schmidt, Phys. Rev. B 87, 054404 (2013).

[13] K. P. Schmidt, Phys. Rev. B 88, 035118 (2013).

[14] K. Coester, W. Malitz, S. Fey, and K. P. Schmidt, Phys. Rev. B 88, 184402 (2013). 
[15] J. Koziol, S. Fey, S. C. Kapfer, and K. P. Schmidt, Phys. Rev. B 100, 144411 (2019)

[16] S. Fey, S. C. Kapfer, and K. P. Schmidt, Phys. Rev. Lett. 122, 017203 (2019).

[17] G. Chen, Phys. Rev. Research 1, 033141 (2019).

[18] R. Coldea, D. A. Tennant, E. M. Wheeler, E. Wawrzynska, D. Prabhakaran, M. Telling, K. Habicht, P. Smeibidl, and K. Kiefer, Science 327, 177 (2010).

[19] A. W. Kinross, M. Fu, T. J. Munsie, H. A. Dabkowska, G. M. Luke, S. Sachdev, and T. Imai, Phys. Rev. X 4, 031008 (2014).

[20] I. Cabrera, J. D. Thompson, R. Coldea, D. Prabhakaran, R. I. Bewley, T. Guidi, J. A. Rodriguez-Rivera, and C. Stock, Phys. Rev. B 90, 014418 (2014).

[21] S. Suga, J. Phys. Soc. Jpn. 77, 074717 (2008).

[22] Z. Wang, T. Lorenz, D. I. Gorbunov, P. T. Cong, Y. Kohama, S. Niesen, O. Breunig, J. Engelmayer, A. Herman, J. Wu, K. Kindo, J. Wosnitza, S. Zherlitsyn, and A. Loidl, Phys. Rev. Lett. 120, 207205 (2018).

[23] Z. Wang, J. Wu, W. Yang, A. K. Bera, D. Kamenskyi, A. T. M. N. Islam, S. Xu, J. M. Law, B. Lake, C. Wu, and A. Loidl, Nature 554, 219 (2018).

[24] Z. He, T. Taniyama, and M. Itoh, Phys. Rev. B 73, 212406 (2006).

[25] Y. Cui, H. Zou, N. Xi, Z. He, Y. X. Yang, L. Shu, G. H. Zhang, Z. Hu, T. Chen, R. Yu, J. Wu, and W. Yu, Phys. Rev. Lett. 123, 067203 (2019).

[26] Y. Shen, C. Liu, Y. Qin, S. Shen, Y.-D. Li, R. Bewley, A. Schneidewind, G. Chen, and J. Zhao, Nat. Commun. 10, 4530 (2019).

[27] F. A. Cevallos, K. Stolze, T. Kong, and R. Cava, Mater. Res. Bull. 105, 154 (2018).

[28] Y. Li, S. Bachus, Y. Tokiwa, A. A. Tsirlin, and P. Gegenwart, arXiv:1804.00696 (2018).

[29] Y. Li, S. Bachus, H. Deng, W. Schmidt, H. Thoma, V. Hutanu, Y. Tokiwa, A. A. Tsirlin, and P. Gegenwart, Phys. Rev. X 10, 011007 (2020).

[30] C. Liu, Y.-D. Li, and G. Chen, Phys. Rev. B 98, 045119 (2018).

[31] P. Bonville, S. Guitteny, A. Gukasov, I. Mirebeau, S. Petit, C. Decorse, M. C. Hatnean, and G. Balakrishnan, Phys. Rev. B 94, 134428 (2016).

[32] G. Chen, Phys. Rev. B 94, 205107 (2016).

[33] Y.-P. Huang, G. Chen, and M. Hermele, Phys. Rev. Lett. 112, 167203 (2014).

[34] Y.-D. Li and G. Chen, Phys. Rev. B 95, 041106(R) (2017).

[35] Y.-D. Li, X. Wang, and G. Chen, Phys. Rev. B 94, 201114(R) (2016).

[36] Y.-D. Li, X. Wang, and G. Chen, Phys. Rev. B 94, 035107 (2016).

[37] E. Lhotel, S. Petit, S. Guitteny, O. Florea, M. Ciomaga Hatnean, C. Colin, E. Ressouche, M. R. Lees, and G. Balakrishnan, Phys. Rev. Lett. 115, 197202 (2015).

[38] V. K. Anand, A. K. Bera, J. Xu, T. Herrmannsdörfer, C. Ritter, and B. Lake, Phys. Rev. B 92, 184418 (2015).

[39] A. Bertin, P. Dalmas de Réotier, B. Fåk, C. Marin, A. Yaouanc, A. Forget, D. Sheptyakov, B. Frick, C. Ritter, A. Amato, C. Baines, and P. J. C. King, Phys. Rev. B 92, 144423 (2015).

[40] J. Xu, V. K. Anand, A. K. Bera, M. Frontzek, D. L. Abernathy, N. Casati, K. Siemensmeyer, and B. Lake, Phys. Rev. B 92, 224430 (2015).
[41] M. C. Hatnean, M. R. Lees, O. A. Petrenko, D. S. Keeble, G. Balakrishnan, M. J. Gutmann, V. V. Klekovkina, and B. Z. Malkin, Phys. Rev. B 91, 174416 (2015).

[42] S. Petit, E. Lhotel, B. Canals, M. C. Hatnean, J. Ollivier, H Mutka, E. Ressouche, A. Wildes, M. Lees, and G. Balakrishnan, Nat. Phys. 12, 746 (2016).

[43] O. Benton, Phys. Rev. B 94, 104430 (2016).

[44] P. Dalmas de Réotier, A. Yaouanc, A. Maisuradze, A. Bertin, P. J. Baker, A. D. Hillier, and A. Forget, Phys. Rev. B 95, 134420 (2017).

[45] C. Mauws, A. M. Hallas, G. Sala, A. A. Aczel, P. M. Sarte, J. Gaudet, D. Ziat, J. A. Quilliam, J. A. Lussier, M. Bieringer, H. D. Zhou, A. Wildes, M. B. Stone, D. Abernathy, G. M. Luke, B. D. Gaulin, and C. R. Wiebe, Phys. Rev. B 98, 100401(R) (2018).

[46] V. Peçanha Antonio, E. Feng, X. Sun, D. Adroja, H. C. Walker, A. S. Gibbs, F. Orlandi, Y. Su, and T. Brückel, Phys. Rev. B 99, 134415 (2019).

[47] R. Sibille, E. Lhotel, V. Pomjakushin, C. Baines, T. Fennell, and M. Kenzelmann, Phys. Rev. Lett. 115, 097202 (2015).

[48] R. Sibille, N. Gauthier, E. Lhotel, V. Porée, V. Pomjakushin, R. A. Ewings, T. G. Perring, J. Ollivier, A. Wildes, C. Ritter et al., Nat. Phys. 16, 546 (2020).

[49] J. Gaudet, E. M. Smith, J. Dudemaine, J. Beare, C. R. C. Buhariwalla, N. P. Butch, M. B. Stone, A. I. Kolesnikov, G. Xu, D. R. Yahne, K. A. Ross, C. A. Marjerrison, J. D. Garrett, G. M. Luke, A. D. Bianchi, and B. D. Gaulin, Phys. Rev. Lett. 122, 187201 (2019).

[50] B. Gao, T. Chen, D. W. Tam, C.-L. Huang, K. Sasmal, D. T Adroja, F. Ye, H. Cao, G. Sala, M. B. Stone, C. Baines, J. A. T. Verezhak, H. Hu, J.-H. Chung, X. Xu, S.-W. Cheong, M. Nallaiyan, S. Spagna, M. Brian Maple, A. H. Nevidomskyy, E. Morosan, G. Chen, and P. Dai, Nat. Phys. 15, 1052 (2019).

[51] X.-P. Yao, Y.-D. Li, and G. Chen, Phys. Rev. Research 2, 013334 (2020).

[52] J. Lago, I. Živković, B. Z. Malkin, J. Rodriguez Fernandez, P. Ghigna, P. Dalmas de Réotier, A. Yaouanc, and T. Rojo, Phys. Rev. Lett. 104, 247203 (2010).

[53] S. Gao, O. Zaharko, V. Tsurkan, L. Prodan, E. Riordan, J. Lago, B. Fåk, A. R. Wildes, M. M. Koza, C. Ritter, P. Fouquet, L. Keller, E. Canévet, M. Medarde, J. Blomgren, C. Johansson, S. R. Giblin, S. Vrtnik, J. Luzar, A. Loidl, C. Rüegg, and T. Fennell, Phys. Rev. Lett. 120, 137201 (2018).

[54] C. Liu, H. Wei, and G. Chen (unpublished).

[55] Yao Shen (private communication).

[56] S. V. Isakov and R. Moessner, Phys. Rev. B 68, 104409 (2003).

[57] Y.-C. Wang, Y. Qi, S. Chen, and Z. Y. Meng, Phys. Rev. B 96, 115160 (2017).

[58] D. Blankschtein, M. Ma, A. N. Berker, G. S. Grest, and C. M. Soukoulis, Phys. Rev. B 29, 5250 (1984).

[59] J. Chen, H.-J. Liao, H.-D. Xie, X.-J. Han, R.-Z. Huang, S. Cheng, Z.-C. Wei, Z.-Y. Xie, and T. Xiang, Chin. Phys. Lett. 34, 050503 (2017).

[60] G. Ortiz, E. Cobanera, and Z. Nussinov, Nucl. Phys. B 854, 780 (2012).

[61] L. Zhang, Phys. Rev. Lett. 123, 230601 (2019).

[62] S. Biswas and K. Damle, Phys. Rev. B 97, 085114 (2018).

[63] K. Damle, Phys. Rev. Lett. 115, 127204 (2015).

[64] J. A. Mydosh and P. M. Oppeneer, Philos. Mag. 94, 3642 (2014). 
[65] A. Smerald and N. Shannon, Phys. Rev. B 88, 184430 (2013).

[66] L. Savary, K. A. Ross, B. D. Gaulin, J. P. C. Ruff, and L. Balents, Phys. Rev. Lett. 109, 167201 (2012).

[67] H. Li, Y.-D. Liao, B.-B. Chen, X.-T. Zen, X.-L. Sheng, Y. Qi, Z. Y. Meng, and W. Li, Nat. Commum. 11, 1111 (2019).

[68] S. Wessel and M. Troyer, Phys. Rev. Lett. 95, 127205 (2005).

[69] R. G. Melko, A. Paramekanti, A. A. Burkov, A. Vishwanath, D. N. Sheng, and L. Balents, Phys. Rev. Lett. 95, 127207 (2005).

[70] C. Liu, F.-Y. Li, and G. Chen, Phys. Rev. B 99, 224407 (2019).

[71] D. Reig-i-Plessis, A. Cote, S. van Geldern, R. D. Mayrhofer, A. A. Aczel, and G. J. MacDougall, Phys. Rev. Materials 3, 114408 (2019).

[72] A. Bertin, Y. Chapuis, P. D. de Réotier, and A. Yaouanc, J. Phys. Condens. Matter 24, 256003 (2012).

[73] T. Yamashita, S. Ohara, and I. Sakamoto, J. Phys. Soc. Jpn. 80, SA080 (2011).

[74] Z.-F. Ding, Y.-X. Yang, J. Zhang, C. Tan, Z.-H. Zhu, G. Chen, and L. Shu, Phys. Rev. B 98, 174404 (2018).
[75] Z. L. Dun, J. Trinh, M. Lee, E. S. Choi, K. Li, Y. F. Hu, Y. X. Wang, N. Blanc, A. P. Ramirez, and H. D. Zhou, Phys. Rev. B 95, 104439 (2017).

[76] Z. L. Dun, J. Trinh, K. Li, M. Lee, K. W. Chen, R. Baumbach, Y. F. Hu, Y. X. Wang, E. S. Choi, B. S. Shastry, A. P. Ramirez, and H. D. Zhou, Phys. Rev. Lett. 116, 157201 (2016).

[77] S. Otsuka and Y. Hinatsu, J. Solid State Chem. 227, 132 (2015).

[78] F.-Y. Li, Y.-D. Li, Y. Yu, A. Paramekanti, and G. Chen, Phys. Rev. B 95, 085132 (2017).

[79] S. Petit, JDN 12, 105 (2011).

[80] D. C. Wallace, Phys. Rev. 128, 1614 (1962).

[81] S. Toth and B. Lake, J. Phys.: Condens. Matter 27, 166002 (2015).

[82] A. L. Chernyshev and M. E. Zhitomirsky, Phys. Rev. B 79, 144416 (2009).

Correction: The Figure 9 caption contained errors in two of the labels indicating figure parts and has been fixed. 